Pacific

Journal of

Mathematics

THE AMBIENT OBSTRUCTION TENSOR AND THE CONFORMAL DEFORMATION COMPLEX

A. Rod Gover and Lawrence J. Peterson 


\title{
THE AMBIENT OBSTRUCTION TENSOR AND THE CONFORMAL DEFORMATION COMPLEX
}

\author{
A. Rod Gover And Lawrence J. Peterson
}

\begin{abstract}
We construct here a conformally invariant differential operator on algebraic Weyl tensors that gives special curved analogues of certain operators related to the deformation complex and that, upon application to the Weyl curvature, yields the (Fefferman-Graham) ambient obstruction tensor. This new definition of the obstruction tensor leads to simple direct proofs that the obstruction tensor is divergence-free and vanishes identically for conformally Einstein metrics. Our main constructions are based on the ambient metric of Fefferman-Graham and its relation to the conformal tractor connection. We prove that the obstruction tensor is an obstruction to finding an ambient metric with curvature harmonic for a certain (ambient) form Laplacian. This leads to a new ambient formula for the obstruction in terms of a power of this form Laplacian acting on the ambient curvature. This result leads us to construct Laplacian-type operators that generalise the conformal Laplacians of Graham-Jenne-Mason-Sparling. We give an algorithm for calculating explicit formulae for these operators, and this is applied to give formulae for the obstruction tensor in dimensions 6 and 8. As background to these issues, we give an explicit construction of the deformation complex in dimensions $n \geq 4$, construct two related (detour) complexes, and establish essential properties of the operators in these.
\end{abstract}

\section{Introduction}

The Bach tensor [1921] has long been considered an important natural invariant in 4-dimensional Riemannian and pseudo-Riemannian geometry and continues to play an interesting role. See [Anderson 2005; Tian and Viaclovsky 2005], for example. It is conformally invariant, vanishes for metrics that are conformal to Einstein metrics, and arises as the total metric variation of the action $\int|C|^{2}$, where

MSC2000: primary 53A55; secondary 22E70, 53A30, 58J10.

Keywords: ambient metric, conformal deformations, conformal geometry, detour complexes, differential complexes.

ARG gratefully acknowledges support from the Royal Society of New Zealand via Marsden Grant no. 02-UOA-108, and from the New Zealand Institute of Mathematics and its Applications for support via a Maclaurin Fellowship. 
$C$ denotes the Weyl curvature. From the latter and the conformal invariance of the Weyl curvature, it follows that it is a symmetric trace-free 2-tensor involving 4 derivatives of the metric. An explicit formula for the Bach tensor in terms of the Weyl curvature $C$, the Ricci tensor, and the Levi-Civita connection is very simple:

$$
B_{a b}=\nabla^{c} \nabla^{d} C_{a c b d}+\frac{1}{2} \operatorname{Ric}^{c d} C_{a c b d} .
$$

In higher even dimensions $n$, an analogue of the Bach tensor was discovered by Fefferman and Graham [1985]; it arose as an obstruction to their ambient metric construction. This Fefferman-Graham obstruction tensor, which we denote $\mathrm{O}_{a b}$ (or sometimes $\mathbb{O}_{a b}^{n}$ ), shares many of the properties of the Bach tensor. It is a trace-free symmetric 2-tensor that vanishes for conformally Einstein metrics. The obstruction tensor has the form $\Delta^{n / 2-2} \nabla^{c} \nabla^{d} C_{a c b d}+$ lots. Here "lots" indicates lower order terms. There is strong evidence that the obstruction tensor will be as important in each even dimension as the Bach tensor is in dimension 4. Very recently Graham and Hirachi [2005] have shown that $O_{a b}$ is the total metric variation of $\int Q$, where $Q$ is Branson's Q-curvature [Branson 1995; Branson and Ørsted 1991]. This generalises the situation in dimension 4 , since in that case $\int Q$ and $\int|C|^{2}$ agree up to a multiple. There is a direct link between the obstruction tensor and the nonexistence of certain operators on conformal manifolds which also generalises the 4-dimensional setting [Gover and Hirachi 2004] and further indicates the critical role of the obstruction tensor.

Despite this progress, the obstruction tensor has remained somewhat mysterious, partly due to the lack of a general formula. In the next section we explain that there is a fundamental difference between the Bach tensor in dimension 4 and the obstruction tensor in even dimensions 6 and greater. The idea is as follows. From the Bianchi identities, the expression (1) for the Bach tensor can be written as

$$
\nabla^{(c} \nabla^{d)} C_{a c b d}+\frac{1}{2} \operatorname{Ric}^{c d} C_{a c b d}
$$

where the parentheses indicate symmetrisation over the index pair $c d$. The differential operator $\nabla^{(c} \nabla^{d)}+\frac{1}{2} \mathrm{Ric}^{c d}$ is a conformally invariant operator which acts on the bundle of "algebraic Weyl tensors" (the bundle whose sections are 4-tensor fields with the same conformal weight and algebraic symmetries as the Weyl curvature) and takes values in a (density weighted) irreducible tensor bundle. One might hope that a similar result would hold in higher dimensions. This is not the case. In Proposition 2.1, we establish that in dimensions $n \geq 6$, the obstruction tensor cannot arise in this manner from a conformally invariant operator that acts between irreducible tensor bundles. This is an easy consequence of representation theory results of Boe and Collingwood [1985] that give a classification of conformally invariant operators on the sphere. (See [Eastwood and Slovák 1997] and references 
therein.) One focus of this article is to describe the correct generalisation of the described construction of the Bach tensor. This is Theorem 2.3, one of the main results.

In the conformally flat setting, the conformally invariant operator defined in the previous paragraph is the formal adjoint of an operator in the so-called (conformal) deformation complex. This is a complex of conformally invariant differential operators arising in connection with infinitesimal deformations of a conformal structure based at a conformally flat metric. The linearisation of the obstruction tensor, which we denote by $\mathrm{B}$, is an operator in a class of conformally invariant operators acting between bundles in the complex. These "long operators" are predicted by the Boe-Collingwood classification. In Proposition 2.2, we show that the linearised obstruction operator and another long operator denoted by $L$ factor through operators from the complex. For example, we obtain that $B=G C$, where $C$ is the linearised Weyl curvature operator and $\mathrm{G}$ is a gauge companion operator for $L$. That is, $L$ and $G$ have the same domain space (algebraic Weyl tensors), the system $(L, G)$ gives a conformally invariant equation, and in Riemannian signature this system is elliptic. Theorem 2.3 gives a curved analogue of this picture. The theorem describes a conformally invariant differential operator $\mathbb{B}$ which, on general conformal manifolds of even dimension, acts on algebraic Weyl tensors and takes values in a reducible bundle. In dimensions $n \geq 6$, composing this with projection to a quotient gives a conformally invariant operator $L$ which takes algebraic Weyl tensors to weighted algebraic Weyl tensors; $L$ generalises $L$ to conformally curved manifolds. This operator annihilates the Weyl curvature $C$, and $\mathbb{B}(C)$ is the obstruction tensor. An application of these results is given in Proposition 2.4, which in the conformally flat setting relates the conformally invariant null space of the system $(L, G)$ to the cohomology of the deformation complex.

For a conformal structure of dimension $n$, the ambient metric is an associated, suitably homogeneous, and Ricci-flat metric on an $(n+2)$-manifold. In [Fefferman and Graham 1985], $O_{a b}$ arose as an obstruction in even dimensions to the existence of a formal power series solution for this ambient metric. In Section 3B, we show that the obstruction tensor may equivalently be viewed as a formal obstruction to having the ambient curvature harmonic for a certain ambient form-Laplacian 4 . This leads to a new proof that the obstruction tensor is an obstruction to the ambient metric (see (v) of Theorem 4.4) and a very simple ambient formula for the obstruction. Let $\boldsymbol{R}$ denote the curvature of the ambient metric. Then $\boldsymbol{\Delta}^{n / 2-2} \boldsymbol{R}$ is a disguised form of the obstruction. This is also established in Theorem 4.4 and in the same place used to give a new proof that the obstruction is divergence-free, i.e. that $\nabla^{a} \mathrm{O}_{a b}=0$. (An alternative proof of this last result is given in [Graham and Hirachi 2005], and it also follows from the variational characterisation given in the same work. See [Branson 2005].) 
An interpretation of these results on the underlying conformal manifold can be achieved via tractor bundles. The standard tractor bundle is a vector bundle with a conformally invariant connection that we may view as arising as an induced structure from the Cartan bundle with its normal conformal Cartan connection. On the other hand, this rank $n+2$ vector bundle also arises in a simple way from the tangent bundle of the ambient manifold. Using this observation, in Theorem 4.1 and Proposition 4.8 we construct families of conformally invariant operators with leading term a power of the Laplacian; these act between arbitrary tractor bundles of an appropriate density weight and generalise the GJMS operators of [Graham et al. 1992]. In Theorem 4.2, we show that the obstruction tensor is obtained by applying one of these operators, namely $\square_{n / 2-2}$, which has the form $\Delta^{n / 2-2}+$ lots, to the tractor field $W$ that corresponds to $\boldsymbol{R}$. Thus the problem of finding formulae for the obstruction tensor is reduced to understanding the special case $\square_{n / 2-2}$ of the generalised GJMS-type operators $\square_{k}$.

There is a one-to-one correspondence between Einstein metrics and a class of parallel standard tractors [Gauduchon 1990; Gover and Nurowski 2006]. With the tractor formula for the obstruction $\square_{n / 2-2} W$, this forms the basis of the proof of Theorem 4.3, which shows that the obstruction vanishes for conformally Einstein metrics.

Theorem 4.1 constructs a very general class of Laplace type conformal operators. The inductive steps leading to Theorem 4.1 yield a simple and effective algorithm for calculating explicit formulae for the conformal Laplacian operators of that theorem. Hence by Theorem 4.2, they give an algorithm for calculating explicit formulae for the obstruction. This algorithm is efficient in the sense that it does not entail constructing the ambient manifold but uses just its existence; the algorithm recovers only those invariants of the ambient metric that actually turn up in the final formula for the operator. In Section 4B, explicit tractor formulae for conformal Laplacian operators are given. See expressions (59) and (62). These are then applied to the $W$-tractor to give formulae for the obstruction in dimensions 6 and 8. Tractor formulae are given in (60) and (64), and formulae in terms of the Levi-Civita connection and its curvature are given in (61) and on page 348.

The next section establishes the basic background and notation before constructing the conformal deformation complex and introducing some related operators. It is a pleasure to thank Tom Branson and Robin Graham for helpful discussions.

\section{Relationship to the conformal deformation complex}

We first sketch here notation and background for conformal structures. Further details may be found in [Čap and Gover 2003], [Gover and Peterson 2003] or [Branson and Gover 2005]. We mainly follow the notational conventions of the 
last of these. Let $M$ be a smooth manifold of dimension $n \geq 3$. To simplify our discussions we assume $M$ is orientable. Recall that a conformal structure on $M$ is a smooth ray subbundle $2 \subset S^{2} T^{*} M$ whose fibre over $x$ consists of conformally related metrics at the point $x$. The principal bundle $\pi: 2 \rightarrow M$ has structure group $\mathbb{R}_{+}$, and so each representation $\mathbb{R}_{+} \ni x \mapsto x^{-w / 2} \in \operatorname{End}(\mathbb{R})$ induces a natural line bundle on $(M,[g])$ that we term the conformal density bundle $E[w]$. We shall write $\mathscr{E}[w]$ for the space of sections of this bundle. Here and throughout the article, sections, tensors, and functions are always smooth. When no confusion is likely to arise, we will use the same notation for a bundle and its section space.

We write $g$ for the conformal metric, the tautological section of $S^{2} T^{*} M \otimes E[2]$ determined by the conformal structure. This will be used to identify $T M$ with $T^{*} M[2]$. For many calculations we will use abstract indices in an obvious way. Given a choice of metric $g$ from the conformal class, we write $\nabla$ for the corresponding Levi-Civita connection. With these conventions the Laplacian $\Delta$ is given by $\Delta=\boldsymbol{g}^{a b} \nabla_{a} \nabla_{b}=\nabla^{b} \nabla_{b}$. Note that $E[w]$ is trivialised by a choice of metric $g$ from the conformal class, and we write $\nabla$ for the connection corresponding to this trivialisation. It follows immediately that (the coupled) $\nabla_{a}$ preserves the conformal metric.

The curvature $R_{a b}{ }^{c} d$ of the Levi-Civita connection is known as the Riemannian curvature and is defined by

$$
\left[\nabla_{a}, \nabla_{b}\right] v^{c}=R_{a b}^{c}{ }^{d} v^{d}
$$

where $[\cdot, \cdot]$ indicates the usual commutator bracket. The Riemannian curvature can be decomposed into the totally trace-free Weyl curvature $C_{a b c d}$ and a remaining part described by the symmetric Schouten tensor $P_{a b}$ using $R_{a b c d}=C_{a b c d}+$ $2 \boldsymbol{g}_{c[a} P_{b] d}+2 \boldsymbol{g}_{d[b} P_{a] c}$, where $[\cdots]$ indicates the antisymmetrisation over the enclosed indices. The Schouten tensor is a trace modification of the Ricci tensor Ric $a b$ and vice versa: writing $J$ for the trace $P_{a}{ }^{a}$ of $P$, then $\operatorname{Ric}_{a b}=(n-2) P_{a b}+J \boldsymbol{g}_{a b}$. Under a conformal transformation we replace a choice of metric $g$ by the metric $\hat{g}=e^{2 \omega} g$, where $\omega$ is a smooth function. Explicit formulae for the corresponding transformation of the Levi-Civita connection and its curvatures are given, for example, in [Bailey et al. 1994a; Gover and Peterson 2003]. We recall that in particular the Weyl curvature is conformally invariant, that is, $\widehat{C}_{a b c d}=C_{a b c d}$.

A tensor $T^{a \cdots b}{ }_{c \cdots d}$ of weight $w$ and with $k$ contravariant indices and $\ell$ covariant indices has total order $\ell-k-w$. For example, the Weyl curvature, the Schouten tensor, and the scalar curvature all have total order 2. The conformal metric $\boldsymbol{g}_{a b}$ has total order zero, and so the total order of any tensor is unchanged by the raising and lowering of indices using the conformal metric. 
A differential operator $P$ is a natural differential operator if it can be written as a universal polynomial in covariant derivatives with coefficients depending polynomially on the metric, its inverse, the curvature tensor, and its covariant derivatives. The coefficients of natural operators are called natural tensors. In the case that they are scalar they are often also called Riemannian invariants. Note that if $T$ is a tensor with total order $t$ then $\nabla T$ has total order $t+1$. It follows immediately that for any natural differential operator $P$ that has $T$ in its domain, the total order of $P T$ is at least $t$. We say $P$ is a conformally invariant differential operator if it is well-defined on conformal structures, i.e., is independent of a choice of conformal scale.

We will use $E^{k}$ as a convenient alternative notation for $\wedge^{k} T^{*} M$. The tensor product of $E^{k} \otimes E^{\ell}, \ell \leq n / 2, k \leq\lceil n / 2\rceil$, decomposes into irreducibles. We denote the highest weight component by $E^{k, \ell}$. (Here "weight" does not refer to conformal weight, but rather to the weight of the inducing $\mathrm{O}(n)$-representation.) We realise the tensors of $E^{k, \ell}$ as trace-free covariant $(k+\ell)$-tensors $T_{a_{1} \cdots a_{k} b_{1} \cdots b_{\ell}}$ which are skew on the indices $a_{1} \cdots a_{k}$ and also on the set $b_{1} \cdots b_{\ell}$. Skewing over more than $k$ indices annihilates $T$, as does symmetrising over any 3 indices. Then we write, for example, $E^{k, \ell}[w]$ as a shorthand for the tensor product $E^{k, \ell} \otimes E[w]$. The space of sections of each of these bundles is indicated by replacing $E$ with $\mathscr{E}$. The sections of $\mathscr{E}^{2,2}$ [2] are the algebraic Weyl tensors as discussed in the introduction, that is, tensors $u_{a b c d}$ with the same symmetries and weight as the Weyl curvature. In particular, the Weyl curvature itself is a section in $\mathscr{E}^{2,2}[2]$. We will also often use the notation $E_{k, \ell}[w]$ as a shorthand for $E^{k, \ell}[w+2 k+2 \ell-n]$. This notation is suggested by the duality between $\mathscr{E}^{k}, \ell[w]$ and $\mathscr{E}_{k, \ell}[-w]$; for $\varphi \in \mathscr{E}^{\mathscr{E}, \ell}[w]$ and $\psi \in \mathscr{E}_{k, \ell}[-w]$, with one of these compactly supported, there is the natural conformally invariant global pairing

$$
\varphi, \psi \mapsto\langle\varphi, \psi\rangle:=\int_{M} \varphi \cdot \psi d \mu_{g},
$$

where $\varphi \cdot \psi \in \mathscr{E}[-n]$ denotes a complete contraction between $\varphi$ and $\psi$.

Since the Weyl curvature is conformally invariant, it follows easily that the linearisation (at a conformally flat metric) of the nonlinear operator $g \mapsto C^{g} \in \mathscr{E}^{2,2}$ [2], with $C^{g}$ the Weyl curvature of the metric $g$, is a conformally invariant operator $C: \mathscr{E}^{1,1}[2] \rightarrow \mathscr{E}^{2,2}[2]$. The formal adjoint of a conformally invariant operator is again conformally invariant. In particular, the formal adjoint of $\mathrm{C}$ is conformally invariant:

$$
\mathrm{C}^{*}: \mathscr{E}_{2,2}[-2] \rightarrow \mathscr{E}_{1,1}[-2] .
$$

Now observe that in dimension 4 we have $\mathscr{E}^{2,2}[2]=\mathscr{E}_{2,2}[-2]$, and so $C^{*}$ acts on the space of algebraic Weyl tensors $\mathscr{E}^{2,2}$ [2]. It is given explicitly up to a multiple by $U_{a b c d} \mapsto\left(\nabla^{(a} \nabla^{c)}+P^{a c}\right) U_{a b c d}$. It is straightforward to verify directly using (34) or the transformation formulae from [Gover and Peterson 2003] that this is 
also conformally invariant in the general curved case, and this operator applied to the Weyl curvature gives the Bach tensor.

On conformally flat structures of dimension at least 4, the null space of $C$ locally agrees with the range of the conformal Killing operator $\mathrm{K}: \mathscr{E}^{1}[2] \rightarrow \mathscr{E}^{1,1}$ [2] given by $v_{a} \mapsto \nabla_{(a} v_{b)_{0}}$, where $(\cdots)_{0}$ indicates the symmetric trace-free part. These operators give the initial sequence of the conformal deformation complex. On oriented structures of dimension 4 this complex is simply

$$
\mathscr{\mathscr { E }} 1[2] \stackrel{\mathrm{K}}{\rightarrow} \mathscr{E} \mathscr{E}^{1,1}[2] \stackrel{\mathrm{C}}{\rightarrow} \mathscr{E}^{2,2}[2] \stackrel{\mathrm{C}^{*} \star}{\rightarrow} \mathscr{E} 1,1[-2] \stackrel{\mathrm{K}^{*}}{\rightarrow} \mathscr{E}_{1}[-2],
$$

where $\star$ is the (conformal) Hodge star operator. Recall that in even dimensions this gives an isomorphism on the space of middle forms $\star: \mathscr{E}^{n / 2} \rightarrow \mathscr{E}^{n / 2}$, and so it also gives an isomorphism $\star: \mathscr{E}^{n / 2,2}[2] \rightarrow \mathscr{E}^{n / 2,2}[2]$.

The situation is more complicated in higher dimensions. In the deformation complex, the operator $C$ is followed by the Weyl-Bianchi operator Bi from $\mathscr{E}^{2,2}$ [2] to $\mathscr{E}^{3,2}$ [2], given (in a conformal scale) by

$$
U_{a b c d} \mapsto(n-3) \nabla_{[a} U_{b c] d e}-g_{d[a} \nabla_{|s|} U_{b c]^{s} e}+g_{e[a} \nabla_{|s|} U_{b c]}^{s} d .
$$

Here $|\cdot|$ indicates that the enclosed indices are omitted from the skew-symmetrisation process. (Note that an easy consequence of its symmetries is that the operator (2) is trivial in dimension 4.) On oriented structures the formal adjoints of these operators conclude the complex, and so we have the picture

$$
\cdot \stackrel{\mathrm{K}}{\rightarrow} \mathscr{E} 1,1[2] \stackrel{\mathrm{C}}{\rightarrow} \mathscr{E}^{2,2}[2] \stackrel{\mathrm{Bi}}{\rightarrow} \mathscr{E}^{3,2}[2] \rightarrow \cdots \rightarrow \mathscr{E}_{3,2}[-2] \stackrel{\overline{\mathrm{Bi}}}{\rightarrow} \mathscr{E}_{2,2}[-2] \stackrel{\mathrm{C}^{*}}{\rightarrow} \mathscr{E}_{1,1}[-2] \stackrel{\mathrm{K}^{*}}{\rightarrow} .
$$

Here we have omitted the initial section space $\mathscr{E}^{1}[2]$ and terminal section space $\mathscr{E}_{1}[-2]$, since they are outside the main focus of our discussions. In dimensions other than $6, \overline{\mathrm{Bi}}$ is $\mathrm{Bi}^{*}$. In dimension $6, \overline{\mathrm{Bi}}$ means the composition $\mathrm{Bi}^{*} \star$. The Hodge star is also implicitly used in interpreting the diagram in dimension 5 . In this case it gives isomorphisms $\star: E^{2,2}[2] \rightarrow E_{3,2}[-2]$ and $\star: E^{3,2}[2] \rightarrow E_{2,2}[-2]$, and under these $\mathrm{Bi}$ is identified modulo a sign with $\mathrm{Bi}^{*}$. In dimensions at least 5 , $\mathrm{C}^{*}$ is given by $U_{a b c d} \mapsto\left(\nabla^{(a} \nabla^{c)}+P^{a c}\right) U_{a b c d}$, the same formula as in dimension 4. In even dimensions $n \geq 8$, the centre of the pattern consists in an obvious way of operators $\mathrm{Bi}_{(k)}: \mathscr{C}_{\mathscr{E}}^{k, 2}[2] \rightarrow \mathscr{C}^{k+1,2}$ [2] for $k=3, \ldots, n / 2-1$, their formal adjoints $\mathrm{Bi}_{(k)}^{*}: \mathscr{E}_{k+1,2}[-2] \rightarrow \mathscr{E}_{k, 2}[-2]$ for $k=3, \ldots, n / 2-2$, and $\mathrm{Bi}_{(n / 2-1)}^{*} \star$ : $\mathscr{E}^{n / 2,2}[2] \rightarrow \mathscr{E}_{n / 2-1,2}[-2]$. The operators $\mathrm{Bi}_{(k)}$ generalise (2), which can be viewed up to a constant multiple as the " $k=2$ case". For $U \in \mathscr{E}^{k, 2}$, an explicit formula is $\left(\operatorname{Bi}_{(k)} U\right)_{a_{0} a_{1} \cdots a_{k} b_{1} b_{2}}=\operatorname{Proj}\left(\nabla_{a_{0}} U_{a_{1} \cdots a_{k} b_{1} b_{2}}\right)$, where Proj is the bundle morphism which executes the projection into $\mathscr{E}^{k+1,2}$ [2]. In odd dimensions at least 7 , we have the operators $\mathrm{Bi}_{(k)}$ for $k=3, \ldots,\lfloor n / 2-1\rfloor$ and their formal adjoints for $k=3, \ldots,\lfloor n / 2-2\rfloor$. (The operator $\mathrm{Bi}_{(\lfloor n / 2-1\rfloor}$ is formally self-adjoint). 
In each dimension, the operators of the deformation complex are all conformally invariant, and the complex is locally exact and extends to give a resolution (on the sheaves of germs of smooth sections) of the sheaf of conformal Killing fields. This is a particular generalised Bernstein-Gelfand-Gelfand (gBGG) resolution. These resolutions are well understood and classified through the dual theory of generalised Verma modules, and the explicit construction of the complex above is an immediate consequence of the local uniqueness of the operators in the relevant gBGG resolution, along with explicit verification of the conformal invariance and nontriviality of the operators mentioned. See [Gasqui and Goldschmidt 1984] for an alternative construction of the complex via a theory of overdetermined systems of partial differential equations based around Spencer cohomology.

According to the results of [Boe and Collingwood 1985], in even dimensions the operators of the deformation complex are not the only conformally invariant operators between the bundles involved. There are also "long operators" from $\mathscr{E}^{k, \ell}[2]$ to $\mathscr{E}_{k, \ell}[-2]$, and an additional pair of operators about the centre of the pattern. We obtain the operator diagram

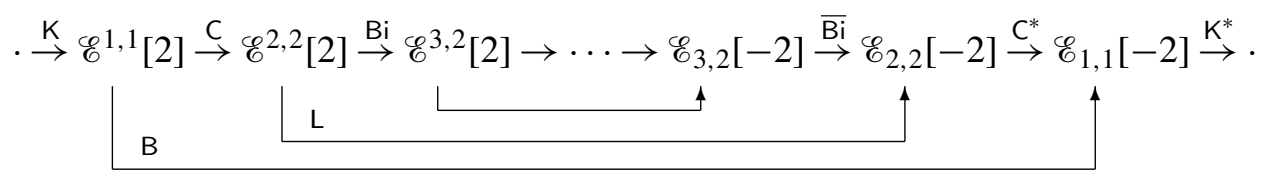

for dimensions 10 or greater. The operators in this diagram are unique up to multiplying by a constant, and the diagram indicates by arrows all the operators between the bundles explicitly presented. Thus, by implication, all compositions vanish. The same diagram applies in dimensions 8 and 6 with minor adjustments. In dimension 8 there are two "short" operators with domain $\mathscr{E}^{3,2}[2]$ and two with range $\mathscr{E}_{3,2}[-2]$. From these there is one nontrivial composition $\mathscr{E}^{3,2}[2] \rightarrow \mathscr{E}_{3,2}[-2]$. Similiarly in dimension 6 we have $\star \mathrm{Bi}: \mathscr{E}^{2,2}[2] \rightarrow \mathscr{E}^{3,2}[2]$ and $\mathrm{Bi}^{*}: \mathscr{E}^{3,2}[2] \rightarrow \mathscr{E}_{2,2}[-2]$, as well as the operators indicated, and $\mathrm{L}=\mathrm{Bi} \mathrm{i}^{*} \mathrm{Bi}$. In dimension 4 the corresponding diagram is

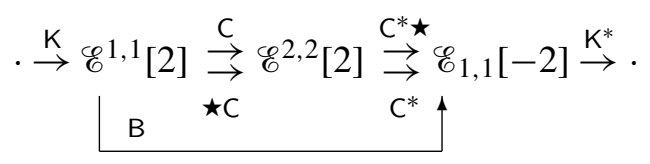

and in this case $B:=C^{*} C$. Evidently on even-dimensional conformally flat structures there are detour complexes [Branson and Gover 2005], where one shortcuts the deformation complex via a long operator. The examples relevant here are

$$
\mathscr{E}^{1}[2] \stackrel{\mathrm{K}}{\rightarrow} \mathscr{\mathscr { E }} 1,1[2] \stackrel{\mathrm{B}}{\longrightarrow} \mathscr{E}_{1,1}[-2] \stackrel{\mathrm{K}^{*}}{\rightarrow} \mathscr{E}_{1}[-2]
$$


and in dimensions $n \geq 6$,

$$
\mathscr{E}^{1}[2] \stackrel{\mathrm{K}}{\rightarrow} \mathscr{\mathscr { E }} 1,1[2] \stackrel{\mathrm{C}}{\rightarrow} \mathscr{E}^{2,2}[2] \stackrel{\mathrm{L}}{\longrightarrow} \mathscr{E}_{2,2}[-2] \stackrel{\mathrm{C}^{*}}{\rightarrow} \mathscr{E}_{1,1}[-2] \stackrel{\mathrm{K}^{*}}{\rightarrow} \mathscr{E}_{1}[-2] .
$$

These have applications in constructing torsion quantities which generalise Cheeger's de Rham half-torsion [Branson and Gover, in progress].

According to [Fefferman and Graham 1985], the obstruction tensor $\mathscr{O}_{a b}$ is a trace-free symmetric 2-tensor of weight $2-n$. In other words, it is a section of $\mathscr{E}_{1,1}[-2]=\mathscr{E}^{1,1}[2-n]$. From the general theory in [Eastwood and Slovák 1997], we know that all the operators indicated explicitly by arrows in the diagrams above admit curved analogues, that is, generalisations to general conformal structures. (In fact, the formulae given above for $\mathrm{K}, \mathrm{C}^{*}$, and $\mathrm{Bi}$ give conformally invariant operators on general structures. We will continue to use this notation for these operators even in the conformally curved setting.) From the diagrams, however, the difference between dimension 4 and higher even dimensions is clear. In dimension 4 there is a conformal operator $\mathscr{E}^{2,2}[2] \rightarrow \mathscr{E}_{1,1}[-2]$ that yields the Bach tensor, as described above. In higher dimensions the conformally invariant $\mathrm{C}^{*}$ does not have $\mathscr{E}^{2,2}[2]$ as its domain. These observations establish the following key point.

Proposition 2.1. In even dimensions $n \geq 6$, there can be no conformally invariant differential operator $\mathscr{E}^{2,2}[2] \rightarrow \mathscr{E}_{1,1}[-2]$ that recovers the obstruction tensor upon application to the Weyl curvature $C$.

If there were such an operator, then by Theorem 4.4 or by [Graham and Hirachi 2005], it would necessarily have as highest order term $\Delta^{n / 2-2} \nabla^{a} \nabla^{c} U_{a b c d}$. Its linearisation would therefore be an operator $\mathscr{E}^{2,2}[2] \rightarrow \mathscr{E}_{1,1}[-2]$. But there is no operator between these bundles in the diagram.

This brings us to the question of whether there can be any conformally invariant operator that yields the obstruction tensor in dimensions $n \geq 6$. We will see that there is, and we will construct the operator. To understand how this works, it is helpful to expose some properties of the operators B and L.

Proposition 2.2. The operators $\mathrm{B}: \mathscr{E}^{1,1}[2] \rightarrow \mathscr{E}_{1,1}[-2]$ and $\mathrm{L}: \mathscr{E}^{2,2}[2] \rightarrow \mathscr{E}_{2,2}[-2]$ are formally self-adjoint. In each even dimension $n \geq 6$, there is a natural linear differential operator $\mathrm{H}: \mathscr{E}^{2,2}[2] \rightarrow \mathscr{E}_{2,2}[-2]$ such that $\mathrm{B}$ is given by the composition

$$
B=C^{*} H C \text {, }
$$

and there is a natural linear differential operator $\mathrm{N}: \mathscr{E}^{3,2}[2] \rightarrow \mathscr{E}_{3,2}[-2]$ such that $\mathrm{L}$ is given by the composition

$$
L=B i^{* N B i} \text {. }
$$

We prove this in Section 4 using the geometric tools developed below. The factorisations described in the proposition can also be established via central character arguments; see also [Branson and Gover 2005]. Note that L is only defined 
in even dimensions $n \geq 6$. In dimension $6, \mathrm{~N}$ is the identity. Otherwise, from the classification of conformally invariant operators on conformally flat manifolds, as discussed above, it follows that the operators $\mathrm{H}$ and $\mathrm{N}$ are not conformally invariant.

On conformally flat structures the operator $\mathrm{G}:=\mathrm{C} * \mathrm{H}$ is not conformally invariant $(n \neq 4)$. It $i s$, however, conformally invariant on the range of the linearised Weyl curvature, and we have $B=G C$. On the other hand, $L$ annihilates the range of $C$. The next theorem gives special curved analogues of these operators.

We need some further notation. On conformal manifolds of dimension $n$ there is a natural reducible, but indecomposable, bundle $W_{2,2}$ that has the composition series $E_{2,2}[-2] \ominus E_{2,1}[-2] \ominus E_{1,1}[-2]$. This means that $E_{1,1}[-2]$ is a (conformally invariant) subbundle and that $E_{2,1}[-2]$ is a subbundle of the quotient $W_{2,2} / E_{1,1}[-2]$. The bundle $W_{2,2}$, which is a subbundle of a certain tractor bundle, is constructed explicitly in proof of Theorem 2.3 in Section 4. It decomposes as $\left[W_{2,2}\right]_{g}=E_{2,2}[-2] \oplus E_{2,1}[-2] \oplus E_{1,1}[-2]$, given a choice of metric $g$ from the conformal class. Let us write $\mathrm{I}^{*}$ and $\mathrm{P}$ for the respective canonical bundle maps $W_{2,2} \rightarrow E_{2,2}[-2]$ and $W_{2,2} \rightarrow E_{2,2}[-2] \ominus E_{2,1}[-2]$ which are unique up to a constant multiple.

Theorem 2.3. On conformal manifolds of even dimension $n \geq 6$ there is a natural nontrivial conformally invariant linear differential operator

$$
\mathbb{B}: \mathscr{E}^{2,2}[2] \rightarrow \mathscr{W}_{2,2}=\mathscr{E}_{2,2}[-2] \oplus \mathscr{E}_{2,1}[-2] \oplus \mathscr{E}_{1,1}[-2]
$$

with the following properties:

(i) The composition $\left(\mathrm{I}^{*} \mathbb{B}=: L\right): \mathscr{E}^{2,2}[2] \rightarrow \mathscr{E}_{2,2}[-2]$ is a nontrivial conformally invariant differential operator of order $n-4$.

(ii) There is a linear differential operator $\mathbb{B}$ such that $\overline{\mathbb{B}} \mathbb{B}=\Delta^{\ell}+$ lots. Thus on Riemannian signature conformal structures, $\mathbb{B}$ is graded injectively elliptic.

(iii) For the Weyl curvature $C \in \mathscr{E}^{2,2}[2]$ we have $\mathbb{B}(C) \in \mathscr{E}_{1,1}[-2]$. The natural conformal invariant $\mathscr{O}_{a b} \in \mathscr{E}_{1,1}[-2]$ given this way agrees with the obstruction tensor.

We prove the theorem in Section 4. Note that there is a degenerate version of the theorem for dimension 4; see expression (28) and the comments that follow it.

From the uniqueness of $L$ it is clear that on conformally flat manifolds $L$ recovers $L$ up to a constant multiple. However $L$ is a special curved generalisation of $L$, since the property $L(C)=0$ generalises to arbitrary conformal structures the vanishing of the composition LC. Since $L(C)$ vanishes, it follows from the composition series for $\mathscr{W}_{2,2}$ that the component of $\mathbb{B}(C)$ in $\mathscr{E}_{2,1}[-2]$ is a natural conformal invariant. That this also vanishes is a special property of $\mathbb{B}$ that, in a sense, carries to general structures the nonexistence of an operator $\mathscr{E}^{1,1}[2] \rightarrow \mathscr{E}_{2,1}[-2]$. It follows that on 
conformally flat structures the composition $\mathbb{B C}$ determines a nontrivial operator $\mathscr{E}^{1,1}[2] \rightarrow \mathscr{E}_{1,1}[-2]$ which therefore agrees with B. If, for each metric $g$ in the conformal class, we write $G$ for the composition of $\mathbb{B}$ followed by projection to the component $\mathscr{E}_{1,1}[-2]$ (we have such a projection since, recall, $\mathscr{W}_{2,2}$ completely decomposes given a conformal scale), then by construction, $G$ is a curved analogue of the operator $\mathrm{G}$. That is, the restriction of $G$ to conformally flat structures is $\mathrm{G}$. Note that $G$ has the special property that $G(C)=\mathbb{O}$, and as we will see from the construction of $W_{2,2}$, although $G$ is not conformally invariant, the conformal variation of $G$ under $g \mapsto e^{2 \omega} g$ is only quadratic in $\omega$. Since $G$ also has this sort of variation, this is optimal.

In the conformally flat case, it is easily shown that $\mathrm{PB}$ can be reexpressed as a composition $\mathbb{U} L$. Here $\mathbb{U}$ is the operator (35) below, except with $w$ set to $6-n$. This result follows from the nonexistence of a nontrivial conformal operator $\mathscr{E}^{2,2}[2] \rightarrow$ $\mathscr{E}_{2,1}[-2]$. It follows from this and (ii) that in even dimensions $n \geq 6,(\mathrm{~L}, \mathrm{G})$ is a right factor of a Laplacian. That is, there are linear differential operators $\bar{L}$ and $\bar{G}$ such that

$$
(\overline{\mathrm{L}}, \overline{\mathrm{G}})\left(\begin{array}{c}
\mathrm{L} \\
\mathrm{G}
\end{array}\right)=\Delta^{\ell}+\text { lots. }
$$

Since also $G$ is conformally invariant on the null space of $L$, it follows that $G$ is a conformal gauge companion operator in the sense of [Branson and Gover 2002]; see also [Branson and Gover 2005]. Thus in Riemannian signature, the operator pair $(L, G)$ is an elliptic system. Since $L$ has $B i$ as a right factor, the system $(B i, G)$ is also elliptic and has a conformally invariant null space. Let us denote this by $\mathscr{H}_{G}^{2}$, and note that on compact manifolds, $\mathscr{H}_{G}^{2}$ is finite-dimensional. This is closely related to the second cohomology of the deformation complex. For example, from Proposition 2.2 and an easy adaption of the proof of Proposition 2.5 in [Branson and Gover 2005], we obtain the following result, which suggests that $\mathscr{H}_{G}^{2}$ is a candidate for a space of conformal harmonics. Here we write $H^{i}, i=1,2$, for the first and second cohomology spaces in the deformation complex, and $H_{B}^{1}$ for the first cohomology of the detour complex (3).

Proposition 2.4. On even-dimensional conformally flat manifolds of dimension $n \geq 6$, there is an exact sequence

$$
0 \rightarrow H^{1} \rightarrow H_{B}^{1} \rightarrow \mathscr{H}_{G}^{2} \rightarrow H^{2},
$$

where the last map $\mathscr{H}_{G}^{2} \rightarrow H^{2}$ is simply $\Phi \mapsto[\Phi]$, the middle $H_{B}^{1} \rightarrow \mathscr{H}_{G}^{2}$ is the map on the quotient $\mathcal{N}(\mathrm{B}) / \mathscr{R}(\mathrm{K})$ induced by the restriction of $\mathrm{C}$ to $\mathcal{N}(\mathrm{B})$, the null space of $\mathrm{B}$, and the first map $H^{1} \rightarrow H_{B}^{1}$ is inclusion.

There are further results concerning the relationship of $H_{B}^{1}$ to $H^{1}$ and $\mathscr{H}_{G}^{2}$ to $H^{2}$, but this will be taken up elsewhere; see also [Branson and Gover 2002]. 


\section{The ambient construction and tractor calculus}

In the subsequent sections we will explore the relationship between the FeffermanGraham ambient metric construction [Fefferman and Graham 1985] and tractor calculus as derived in [Čap and Gover 2003; Gover and Peterson 2003]. The notation and conventions for the ambient metric closely follow [Branson and Gover 2005].

For $\pi: 2 \rightarrow M$ a conformal structure of signature $(p, q)$, use $\rho$ to denote the $\mathbb{R}_{+}$ action on 2 given by $\rho(s)\left(x, g_{x}\right)=\left(x, s^{2} g_{x}\right)$. An ambient manifold is a smooth $(n+2)$-manifold $\tilde{M}$ endowed with a free $\mathbb{R}_{+}$-action $\rho$ and an $\mathbb{R}_{+}$-equivariant embedding $i: 2 \rightarrow \tilde{M}$. We write $\boldsymbol{X} \in \mathfrak{X}(\tilde{M})$ for the fundamental field generating the $\mathbb{R}_{+}$-action: for $f \in C^{\infty}(\tilde{M})$ and $u \in \tilde{M}$, we have $\boldsymbol{X} f(u)=\left.(d / d t) f\left(\rho\left(e^{t}\right) u\right)\right|_{t=0}$. For an ambient manifold $\tilde{M}$, an ambient metric is a pseudo-Riemannian metric $\boldsymbol{h}$ of signature $(p+1, q+1)$ on $\tilde{M}$ satisfying the conditions: (i) $\mathscr{L}_{\boldsymbol{X}} \boldsymbol{h}=2 \boldsymbol{h}$, where $\mathscr{L}_{\boldsymbol{X}}$ denotes the Lie derivative by $\boldsymbol{X}$; (ii) for $u=\left(x, g_{x}\right) \in 2$ and $\xi, \eta \in T_{u} 2$, we have $\boldsymbol{h}\left(i_{*} \xi, i_{*} \eta\right)=g_{x}\left(\pi_{*} \xi, \pi_{*} \eta\right)$; (iii) $\operatorname{Ric}(\boldsymbol{h})=0$ up to the addition of terms vanishing to order $n / 2-1$ if $n$ is even or $\operatorname{Ric}(\boldsymbol{h})=0$ to all orders if $n$ is odd; and (iv) $\boldsymbol{h}(\boldsymbol{X}, \cdot)=\frac{1}{2} d Q$ to all orders.

If $M$ is locally conformally flat, then there is a canonical solution to the ambient metric problem to all orders. This is simply a flat ambient metric. This is forced by (i)-(iii) in odd dimensions, but in even dimensions this extends the solution; see comments in [Branson and Gover 2005]. When discussing the conformally flat case, we assume this solution.

We write $\nabla$ for the ambient Levi-Civita connection and use uppercase abstract indices $A, B$, etc., for tensors on $\tilde{M}$. The ambient Riemann tensor will be denoted $\boldsymbol{R}_{A B}{ }^{C}{ }_{D}$. Since $\mathscr{L}_{\boldsymbol{X}} \boldsymbol{h}=2 \boldsymbol{h}$, it follows that

$$
\begin{gathered}
\nabla \boldsymbol{X}=\boldsymbol{h}, \\
\boldsymbol{X}^{A} \boldsymbol{R}_{A B C D}=0 .
\end{gathered}
$$

Equalities without qualification, as here, indicate that the results hold to all orders or identically on the ambient manifold.

3A. Tractor bundles. Let $\tilde{\mathscr{E}}(w)$ denote the space of functions on $\tilde{M}$ which are homogeneous of degree $w \in \mathbb{R}$ with respect to the action $\rho$. More generally, a tensor field $F$ on $\tilde{M}$ is said to be homogeneous of degree $w$ if $\rho(s)^{*} F=s^{w} F$, that is, $\mathscr{L}_{X} F=w F$. Just as sections of $\mathscr{E}[w]$ are equivalent to functions in $\left.\tilde{\mathscr{E}}(w)\right|_{2}$, the restriction of a homogeneous tensor field to 2 has an interpretation on $M$. Denote by $\mathcal{T}$ the space of sections of $T \tilde{M}$ which are homogeneous of degree -1 and write $\mathcal{T}(w)$ for sections in $\mathcal{T} \otimes \tilde{\mathscr{E}}(w)$, where here $\otimes$ indicates a tensor product over $\tilde{\mathscr{E}}(0)$. From [Čap and Gover 2003] we have the following results: We may identify the 
standard tractor bundle $\mathbb{T}$ with $\left.T \tilde{M}\right|_{2}$ modulo a suitable $\mathbb{R}_{+}$-action, so that sections of $\mathbb{T}$ are in one-one correspondence with sections in $\mathcal{T}$. Thus we write $\mathscr{T}$ for the space of sections of the standard tractor bundle. The filtration of $\mathbb{T}$, which we traditionally indicate by a composition series,

$$
\mathbb{T}=E[1] \ominus E^{1}[1] \ominus E[-1],
$$

reflects the vertical subbundle of $T 2$ and $T 2$ as a subbundle of $\left.T \tilde{M}\right|_{2}$. Then since the ambient metric $\boldsymbol{h}$ is homogeneous of degree 2, it descends to give a metric on $\mathbb{T}$. This is the usual tractor metric. Sections of $\mathcal{T}$ may be characterised as those sections of $T \tilde{M}$ which are covariantly parallel along the integral curves of $\boldsymbol{X}$, which on 2 are exactly the fibres of $\pi$. The normal tractor connection agrees with the ambient connection as follows. For $U \in \mathcal{T}$, let $\tilde{U}$ be the corresponding section of $\left.\mathcal{T}\right|_{2}$. A tangent vector field $\xi$ on $M$ has a lift to a field $\tilde{\xi} \in \mathcal{T}(1)$ on 2 , which is everywhere tangent to 2. This is unique up to adding $f \boldsymbol{X}$, where $f \in \tilde{\mathscr{E}}(0)$. We extend $\tilde{U}$ and $\tilde{\xi}$ smoothly and homogeneously to fields on $\tilde{M}$ and form $\left.\nabla_{\tilde{\xi}} \tilde{U}\right|_{2}$; this section is independent of the extensions and independent of the choice of $\tilde{\xi}$ as a lift of $\xi$ and is exactly the section of $\left.\mathcal{T}(0)\right|_{2}$ corresponding to $\nabla_{\xi} U$ where $\nabla$ here indicates the tractor connection.

When abstract indices are required, the section spaces of the tractor bundle and its dual can also be denoted $\mathscr{T}^{A}$ and $\mathscr{T}_{A}$. A choice of metric $g$ from the conformal class determines a canonical splitting of the composition series (6), by [Bailey et al. 1994a; Čap and Gover 2000]. Via this splitting, direct sums $\oplus$ replace the semidirect sums $\theta$ in that series, and we introduce $g$-dependent sections $Z^{A b}$ in $\mathscr{T}^{A b}[-1]$ and $Y^{A}$ in $\mathscr{T}^{A}[-1]$ that describe the decomposition of $\mathbb{T}$ into the direct $\operatorname{sum} E[1] \oplus E_{a}[1] \oplus E[-1]$. According to $V^{A}=Y^{A} \sigma+Z^{A b} \mu_{b}+X^{A} \rho$, a section $V \in \mathcal{T}$ then corresponds to a triple $[V]_{g}=(\sigma, \mu, \rho)$ of sections, and in these terms the tractor metric is given by $h(V, V)=g^{a b} \mu_{a} \mu_{b}+2 \sigma \rho$. Thus the tractor contractions of the projectors are

$$
X^{A} Y_{A}=1, \quad Z^{A b} Z_{A a}=\delta_{a}^{b},
$$

and 0 for the other pairings.

If $\hat{Y}^{A}$ and $\hat{Z}_{b}^{A}$ are the projectors for the metric $\hat{g}=e^{2 \omega} g$, then we have

$$
\hat{Z}^{A b}=Z^{A b}+\Upsilon^{b} X^{A}, \quad \hat{Y}^{A}=Y^{A}-\Upsilon_{b} Z^{A b}-\frac{1}{2} \Upsilon_{b} \Upsilon^{b} X^{A} .
$$

Here $\Upsilon:=d \omega$. In terms of this splitting, determined by $g$, the tractor connection is given by

$$
\nabla_{a} X_{A}=Z_{A a}, \nabla_{a} Z_{A b}=-P_{a b} X_{A}-Y_{A} \boldsymbol{g}_{a b} \text {, and } \nabla_{a} Y_{A}=P_{a b} Z_{A}^{b} .
$$

We use the notation $\tilde{\mathbb{T}}^{\Phi}$ to denote an arbitrary ambient tensor bundle (with $\tilde{\mathbb{T}}^{0}$ meaning the trivial bundle) and write $\mathcal{T}^{\Phi}(w), w \in \mathbb{R}$, for the subspace of $\Gamma\left(\tilde{\mathbb{T}}^{\Phi}\right)$ 
consisting of sections $S$ satisfying $\nabla_{X} S=w S$; we will say such sections are homogeneous of weight $w$. From the constructions above, it follows that the sections in $\left.\mathcal{T}^{\Phi}(w)\right|_{2}$ are equivalent to sections of a tractor bundle that we denote $\mathbb{T}^{\Phi}[w]$. We write $\mathscr{T}^{\Phi}[w]$ for the section space of the latter.

A basic example of interest is the bundle of $k$-form tractors $\mathbb{T}^{k}$, which is the $k$-th exterior power of the bundle of standard tractors. It is straightforward to verify that this has a composition series which, in terms of section spaces, is given by

$$
\mathscr{T}^{k}=\Lambda^{k} \mathfrak{T} \cong \mathscr{E}^{k-1}[k] \ominus\left\{\mathscr{E}^{k}[k] \oplus \mathscr{E}^{k-2}[k-2]\right\} \bullet \mathscr{E}^{k-1}[k-2] .
$$

Also of direct relevance to our constructions below are the bundles denoted by $\mathbb{T}^{2,2}[w]$, which are the subbundles of $\mathbb{T}^{2} \otimes \mathbb{T}^{2} \otimes E[w]$ consisting of tractors of weight $w$ and Weyl-tensor-type symmetries, that is, trace-free Riemann-tensortype symmetries. We write $\mathscr{T}^{2,2}[w]$ for the section space of $\mathbb{T}^{2,2}[w]$ and note that (with notation as in Section 2) it has the composition series

$$
\begin{aligned}
& \mathscr{E}^{2,2}[w+4] \\
& \oplus \\
& \mathscr{E}^{2,1}[w+4] \quad \mathscr{E}^{2}[w+2] \quad \mathscr{E}^{2,1}[w+2]
\end{aligned}
$$

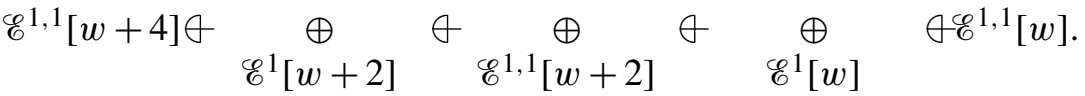

$$
\begin{aligned}
& \oplus \\
& \mathscr{E}[w]
\end{aligned}
$$

A comment on punctuation is in order: here the columns represent composition factors, decomposed into $O(g)$-irreducibles, and these are separated by $\theta$ 's which indicate the composition structure. This series may be obtained by any $s o(n+2)$ to $\mathfrak{s o}(n)$ branching-rule algorithm or, alternatively, by simply considering the possible contractions of the projectors $X, Y$, and $Z$ into a typical element of $\mathscr{T}^{2,2}[w]$.

3B. Operators and invariants via the ambient metric. An operator $P$ acting between ambient tensor bundles is said to be homogeneous of weight $u \in \mathbb{R}$ if $\left[\nabla_{X}, P\right]=u P$. Operators homogeneous in this sense map homogeneous tensors of weight $w$ to homogeneous tensors of weight $w+u$. On the other hand, a differential operator $P$ is said to act tangentially along 2 , as an operator on some domain space, if we have $P Q=Q P^{\prime}$ for some operator $P^{\prime}$ (or equivalently $[P, Q]=Q P^{\prime \prime}$ for some $P^{\prime \prime}$ ). Of particular interest are linear differential operators $P$ which are both homogeneous and also act on some homogeneous tensor space $\mathcal{T}^{\Phi}(w)$ as domain tangentially along 2 . Each such operator $P$ clearly determines a welldefined operator on $\left.\mathcal{T}^{\Phi}(w)\right|_{2}$, and hence determines an operator on the equivalent weighted tractor bundle section space $\mathscr{T}^{\Phi}[w]$. If the operator $P$ is natural as an operator on the ambient manifold, then since the ambient construction is not dependent on a choice of metric from the conformal class, it follows that the induced 
operator on weighted tractor fields is conformally invariant. The remaining issue is whether this induced operator is natural for the underlying conformal structure. For the operators we are interested in here, we solve this by giving an algorithm for expressing the induced operator as a formula in terms of known natural operators. This solves two problems, since one of our aims is to obtain explicit formulae for the operators concerned.

Before we construct examples of such operators, we require some further background. First note that from (4), we have

$$
[\boldsymbol{\Delta}, \boldsymbol{X}]=2 \nabla, \quad \text { where } \quad \boldsymbol{\Delta}:=\nabla^{A} \nabla_{A},
$$

and $\nabla_{A} Q=2 X_{A}$. Both identities hold to all orders. Thus $\nabla_{X} Q=2 Q ; Q$ is homogeneous of weight 2. A short computation shows that if $U$ is an ambient tensor field, then

$$
[\Delta, Q] U=2\left(n+2 \nabla_{X}+2\right) U .
$$

It follows that for any positive integer $\ell$, if an ambient tensor field $U$ is $O\left(Q^{\ell}\right)$, then $\Delta U$ and $\nabla U$ are both $O\left(Q^{\ell-1}\right)$.

Now we define an operator $\boldsymbol{D}$ (or $\boldsymbol{D}_{A}$ when indices are used). Let

$$
D V:=\nabla\left(n+2 \nabla_{X}-2\right) V-X \boldsymbol{\Delta} V,
$$

for any ambient tensor field $V$. It is readily verified that $\boldsymbol{D}$ is homogeneous of weight -1 . By (12) we also have the equivalent formula

$$
\boldsymbol{D} V=\nabla\left(n+2 \nabla_{X}\right) V-\Delta X V .
$$

Using either of these with the computations above, we obtain $D Q V=Q D V+$ $4 Q \nabla V$, and so $\boldsymbol{D}$ acts tangentially. For later use we note that for any integer $\ell \geq 2$, if $V$ is $O\left(Q^{\ell}\right)$, then $\boldsymbol{D}_{A} V$ is $O\left(Q^{\ell-1}\right)$.

Since $\boldsymbol{D}$ acts tangentially on any ambient tensor bundle, it follows that for every tractor bundle $\mathscr{T}^{\Phi}$ and $w \in \mathbb{R}$ we obtain an operator

$$
D: \mathscr{T}^{\Phi}[w] \rightarrow \mathscr{T} \otimes \mathscr{T}^{\Phi}[w-1]
$$

equivalent to $\boldsymbol{D}$ as an operator $\left.\left.\boldsymbol{T}^{\Phi}(w)\right|_{2} \rightarrow \boldsymbol{T} \otimes \mathcal{T}^{\Phi}(w-1)\right|_{2}$. It is straightforward to prove (see [Čap and Gover 2003; Gover and Peterson 2003]) that $D$ is the usual tractor-D operator of [Thomas 1926; Bailey et al. 1994a]. For a choice of metric $g$ from the conformal class and for any $V \in \mathscr{T}^{\Phi}[w], D$ is given explicitly by

$$
D^{A} V:=(n+2 w-2) w Y^{A} V+(n+2 w-2) Z^{A a} \nabla_{a} V-X^{A} \square V,
$$

where $\square V:=\Delta V+w J V$. We note that $D$ is a natural differential operator. A differential operator taking values in a tractor bundle (or acting between tractor 
bundles) is said to be natural if the $\mathfrak{s o}(g)$-irreducible components of the operator are natural.

Acting on $\boldsymbol{T}^{\Phi}(1-n / 2)$, the operator $\boldsymbol{D}$ is simply $-\boldsymbol{X} \boldsymbol{\Delta}$, and correspondingly $D$ simplifies to $-X \square$ on $\mathscr{T}^{\Phi}[1-n / 2]$. Thus $\boldsymbol{\Delta}$ acts tangentially on $\mathcal{T}^{\Phi}(1-n / 2)$ and as an operator on the restriction of this space to 2 , is equivalent to the tractorcoupled conformal Laplacian

$$
\square: \mathscr{T}^{\Phi}[1-n / 2] \rightarrow \mathscr{T}^{\Phi}[-1-n / 2] .
$$

Many identities involving $D$ are obtained most easily by calculating with $\boldsymbol{D}$ on $\tilde{M}$. For example, a short calculation using (4) and (12) shows that

$$
\boldsymbol{D}_{A} \boldsymbol{X}^{A} V=(n+2 w+2)(n+w) V-Q \boldsymbol{\Delta} V
$$

for any $V \in \mathcal{T}^{\Phi}(w)$. Hence for any $V \in \mathscr{T}^{\Phi}[w]$, we have

$$
D_{A} X^{A} V=(n+2 w+2)(n+w) V .
$$

An observation key to the next section is that the ambient curvature $\boldsymbol{R}$ is "harmonic" for a certain Laplacian, at least at low orders. Before we construct this Laplacian we need some further notation. Write $\sharp$ (hash) for the natural tensorial action of sections $A$ of $\operatorname{End}(T \tilde{M})$ on ambient tensors. For example, on an ambient covariant 2-tensor $T_{A B}$, we have $A \sharp T_{A B}=-A^{C}{ }_{A} T_{C B}-A^{C}{ }_{B} T_{A C}$. If $A$ is skew for $\boldsymbol{h}$, then at each point, $A$ is $\mathfrak{s o}(\boldsymbol{h})$-valued. The hash action thus commutes with the raising and lowering of indices and preserves the $\mathrm{SO}(\boldsymbol{h})$-decomposition of tensors. For example, $A \sharp$ maps trace-free symmetric tensors to trace-free symmetric tensors. As a section of the tensor square of the $\boldsymbol{h}$-skew bundle endomorphisms of $T \tilde{M}$, the ambient curvature has a double hash action on ambient tensors; we write $\boldsymbol{R} \sharp \sharp T$. As a point on punctuation, it should be noted that we will treat tensors in composite expressions as multiplication operators. A composition of operators $L$, $M$, and $N$ acting on $S$ denoted $L M N S$ means $L(M(N(S)))$. For example, $\nabla \boldsymbol{R} \sharp \sharp T$ has the same interpretation as $\nabla(\boldsymbol{R} \sharp \sharp T)$.

From the Bianchi identities, we have that on any Riemannian or pseudo-Riemannian manifold,

$$
\begin{aligned}
& 4 \nabla_{A_{1}} \nabla_{B_{1}} \boldsymbol{R i c}_{A_{2} B_{2}} \\
& =\boldsymbol{\Delta} \boldsymbol{R}_{A_{1} A_{2} B_{1} B_{2}}+\frac{1}{2} \boldsymbol{R} \sharp \sharp \boldsymbol{R}_{A_{1} A_{2} B_{1} B_{2}}-\boldsymbol{R i c}_{C A_{1}} \boldsymbol{R}^{C}{ }_{A_{2} B_{1} B_{2}}+\boldsymbol{R i c}_{C B_{1}} \boldsymbol{R}_{B_{2} A_{1} A_{2}} .
\end{aligned}
$$

Remark. In (20) we adopt the convention that sequentially labeled indices in the subscript position (such as $A_{1}$ and $A_{2}$ ) are implicitly skew-symmetrised. This convention applies throughout this paper unless noted otherwise. 
Let us define a Laplacian operator $\Delta$ by the formula

$$
\boldsymbol{\Delta}:=\boldsymbol{\Delta}+\frac{1}{2} \boldsymbol{R} \sharp \sharp \text {. }
$$

Then from (20) and the conditions on $\operatorname{Ric}(\boldsymbol{h})$ for the ambient metric, we have

$$
4 \nabla_{A_{1}} \nabla_{B_{1}} \mathbf{R i c}_{A_{2} B_{2}}=\Delta \boldsymbol{R}_{A_{1} A_{2} B_{1} B_{2}}+O\left(Q^{n / 2-1}\right)
$$

in even dimensions. Therefore

$$
\triangle \boldsymbol{R}_{B C D E}=0
$$

modulo $O\left(Q^{n / 2-3}\right)$ in even dimensions and to infinite order in odd dimensions.

Remarks. 1. The operator $\Delta$ is a type of form-Laplacian. On a Riemannian or pseudo-Riemannian manifold, suppose $U$ is any tensor with Riemann tensor type symmetries. A short calculation shows that

$$
\Delta U=-\frac{1}{2}\left(\delta_{1}^{\nabla} d_{1}^{\nabla}+d_{1}^{\nabla} \delta_{1}^{\nabla}+\delta_{2}^{\nabla} d_{2}^{\nabla}+d_{2}^{\nabla} \delta_{2}^{\nabla}\right) U,
$$

where $\boldsymbol{d}_{\boldsymbol{i}}^{\nabla}$ is the Levi-Civita connection-coupled exterior derivative, $\boldsymbol{\delta}_{\boldsymbol{i}}^{\nabla}$ is its formal adjoint, and the index $i$ is 1 or 2 according to whether we regard $U$ as a 2-form with values in a tensor bundle on the first pair of indices or the last pair. In terms of the Levi-Civita connection $\nabla$, we have $\left(\boldsymbol{d}_{1}^{\nabla} U\right)_{A_{0} A_{1} A_{2} B_{1} B_{2}}=3 \nabla_{A_{0}} U_{A_{1} A_{2} B_{1} B_{2}}$ and $\left(\boldsymbol{\delta}_{\mathbf{2}}^{\nabla} U\right)_{A_{1} A_{2} B_{2}}=-\nabla^{B_{1}} U_{A_{1} A_{2} B_{1} B_{2}}$, for example.

Returning to the ambient manifold, note that from these observations, the results concerning the degree to which the ambient curvature is $\Delta$-harmonic are manifest, since on the one hand $\boldsymbol{d}_{\mathbf{1}}^{\nabla}$ and $\boldsymbol{d}_{\mathbf{2}}^{\nabla}$ annihilate $\boldsymbol{R}$ by the Bianchi identity and on the other hand $\delta_{1}^{\nabla} \boldsymbol{R}$ and $\delta_{2}^{\nabla} \boldsymbol{R}$ are $O\left(Q^{n / 2-2}\right)$ (or $O\left(Q^{\infty}\right)$ in odd dimensions) by dint of the contracted Bianchi identity and the condition (iii) on the ambient Ricci curvature.

2. Note that from (20), if Ric vanishes to all orders on the ambient manifold, then it is immediate that $\boldsymbol{\Delta} \boldsymbol{R}$ vanishes to all orders. Conversely, if $\boldsymbol{\Delta} \boldsymbol{R}$ vanishes to all orders, then so does $4 \nabla_{A_{1}} \nabla_{B_{1}} \boldsymbol{R i c}_{B_{2} A_{2}}+\boldsymbol{R i c}_{C A_{1}} \boldsymbol{R}^{C}{ }_{A_{2} B_{1} B_{2}}-\boldsymbol{R i c}_{C B_{1}} \boldsymbol{R}_{B_{2} A_{1} A_{2}}$. On the other hand, contracting the latter with $\boldsymbol{X}^{A_{1}} \boldsymbol{X}^{B_{1}}$ and using (4) and (5) yields $2 \mathbf{R i c}_{A_{2} B_{2}}$. Thus on the ambient manifold, the vanishing of Ric to all orders is equivalent to the vanishing of $\boldsymbol{\Delta} \boldsymbol{R}$ to all orders.

We may view the operator 4 as the special case $\alpha=\frac{1}{2}$ of the family of ambient Laplacians

$$
\boldsymbol{\Delta}_{\alpha}:=\boldsymbol{\Delta}+\alpha \boldsymbol{R} \sharp \sharp, \quad \alpha \in \mathbb{R},
$$

which also includes the ambient form Laplacian at $\alpha=1$ and the usual ambient Bochner Laplacian at $\alpha=0$. While the latter was used in the constructions of 
[Graham et al. 1992] giving conformal operators between densities, the generalisation to the ambient form Laplacian proved appropriate in [Branson and Gover 2005] for the study of conformal operators on (weighted) differential forms. It seems likely that others in the family will also have important roles, and so much of the discussion in the next section allows for the possibility of any $\alpha \in \mathbb{R}$. Certain key identities for $\boldsymbol{\Delta}$ are unaffected by the addition of the $\boldsymbol{R} \sharp \sharp$ term. In particular, since $\boldsymbol{X}^{A} \boldsymbol{R}_{A B C D}=0$ it follows that

$$
\left[\boldsymbol{\Delta}_{\alpha}, \boldsymbol{X}\right]=[\boldsymbol{\Delta}, \boldsymbol{X}]=2 \nabla .
$$

Using this, or even more simply by noting that $[\boldsymbol{R} \sharp \sharp, Q]=0$, we obtain

$$
\left[\boldsymbol{\Delta}_{\alpha}, Q\right]=[\boldsymbol{\Delta}, Q]=2\left(n+2 \nabla_{\boldsymbol{X}}+2\right) .
$$

A point of departure is $\left[\boldsymbol{\Delta}_{\alpha}, \nabla\right]$. Observe that if $V_{B C \cdots E}$ is any ambient tensor, then by the Ricci-flatness of the ambient metric,

$$
\begin{aligned}
& {\left[\boldsymbol{\Delta}, \nabla_{A}\right] V_{B C \cdots E}} \\
& =-2 \boldsymbol{R}_{A}{ }^{P}{ }_{B}{ }^{Q} \nabla_{P} V_{Q C \cdots E}-2 \boldsymbol{R}_{A}{ }^{P}{ }_{C}{ }^{Q} \nabla_{P} V_{B Q \cdots E}-\cdots-2 \boldsymbol{R}_{A}{ }^{P}{ }_{E}{ }^{Q} \nabla_{P} V_{B C \cdots Q} .
\end{aligned}
$$

This equality holds modulo $O\left(Q^{n / 2-2}\right)$ in even dimensions and to infinite order in odd dimensions.

Using the results above and the Bianchi identities, it is straightforward to verify that if we define the ambient homogeneous (of weight -2) tensor field

$$
\boldsymbol{W}_{A_{1} A_{2} B_{1} B_{2}}:=\frac{3}{n-2} \boldsymbol{D}^{A_{0}} \boldsymbol{X}_{A_{0}} \boldsymbol{R}_{A_{1} A_{2} B_{1} B_{2}},
$$

then in dimensions other than 4 , we have $\left.\boldsymbol{W}\right|_{2}=\left.(n-4) \boldsymbol{R}\right|_{2}$. Note that $\boldsymbol{W}$ is well-defined in all dimensions and by construction is conformally invariant. Thus the equivalent tractor field $W_{A B C E}$ is conformally invariant and of weight -2 . In dimensions other than 4 , it is immediate that this has Weyl tensor type symmetries. (Recall that $\left.\boldsymbol{R}\right|_{2}$ is trace-free.) In fact, it has these symmetries in all dimensions and is a natural tractor field. In a choice of conformal scale, $W_{A B C E}$ is given by

$$
\begin{aligned}
(n-4)\left(Z_{A}{ }^{a} Z_{B}{ }^{b} Z_{C}{ }^{c} Z_{E}{ }^{e} C_{a b c e}-2 Z_{A}{ }^{a} Z_{B}{ }^{b} X_{[C} Z_{E]}{ }^{e} A_{e a b}\right. \\
\left.-2 X_{[A} Z_{B]}^{b} Z_{C}{ }^{c} Z_{E}^{e} A_{b c e}\right)+4 X_{[A} Z_{B]}^{b} X_{[C} Z_{E]}^{e} B_{e b},
\end{aligned}
$$

where $A_{a b c}$ is the Cotton tensor,

$$
A_{a b c}:=2 \nabla_{[b} P_{c] a},
$$

and

$$
B_{a b}:=\nabla^{c} A_{a c b}+P^{d c} C_{d a c b} .
$$


Note that from (28) it follows that in dimension $4, B_{e b}$ is conformally invariant. This is the Bach tensor: from the contracted Bianchi identity, we have

$$
(n-3) A_{a b c}=\nabla^{d} C_{d a b c},
$$

and so in dimension 4, (30) agrees with (1). In other dimensions $n \geq 3$ we also refer to $B_{a b}$ as defined in (30) as the Bach tensor. The tractor field $W$ first appeared in [Gover 1999, 2001]. The connection to the ambient curvature was derived in [Čap and Gover 2003], where the above results are treated in detail.

\section{Conformal Laplacians and the ambient obstruction}

In this section we show how one can obtain the ambient obstruction tensor by applying a conformally invariant operator $\square_{n / 2-2}$ of the form $\Delta^{n / 2-2}+$ lots to the natural tractor field $W$ defined above. For any integer $m \geq 1$, we let

$$
\square_{m}:=\square_{m}^{1 / 2},
$$

where $\square_{m}^{1 / 2}$ is the case $\alpha=1 / 2$ of the operator $\square_{m}^{\alpha}$ of Theorem 4.1. We prove Theorem 4.1 in Section 4A. The inductive nature of this proof will show that one can construct explicit tractor formulae for the operators $\square_{m}^{\alpha}$ in terms of $X, D, W$, $h$, and $h^{-1}$. One may thus use Theorem 4.2 together with a choice of conformal scale and the formula for $W$ given in (28) to construct a tractor formula for $\mathrm{O}_{a b}$. It is then easy to expand this tractor formula to a formula in terms of the Levi-Civita connection and its curvature.

In what follows, the phrase "generic $n$-even case" refers to the case in which $n$ is even and $M$ is conformally curved.

Theorem 4.1. For every integer $m \geq 1$ and for every $\alpha \in \mathbb{R}$, there exists a conformally invariant operator $\square_{m}^{\alpha}: \mathscr{T}^{\Phi}[m-n / 2] \rightarrow \mathscr{T}^{\Phi}[-m-n / 2]$ having leading term $\Delta^{m}$. The operator $\square_{m}^{\alpha}$ is natural in the following cases: in odd dimensions and for conformally flat $M$ for all $m \geq 1$; in the generic n-even case for $1 \leq m \leq n / 2-2$, if $\alpha=0$ for $1 \leq m \leq n / 2-1$, if $\mathscr{T}^{\Phi}[m-n / 2]=\mathscr{T}[m-n / 2]$ for $1 \leq m \leq n / 2-1$, or if $\mathscr{T}^{\Phi}[m-n / 2]=\mathscr{T}^{0}[m-n / 2]$ for $1 \leq m \leq n / 2$. In these cases there is a tractor formula for $\square_{m}^{\alpha}$ given by a partial contraction polynomial in $\square, D, W, X, h$, and $h^{-1}$, and this polynomial is linear in $U$. In the tractor formula for $\square_{m}^{\alpha} U$, each free index appears either on $U$ or on a $W$-tractor.

We believe the operators $\square_{m}$ will be important for many problems. For our current purposes, we are primarily interested in them when $n$ is even, $m=n / 2-2$, and the domain bundle is $\mathscr{T}^{2,2}[-2]$. In particular, we have the following result, which is an immediate consequence of Theorem 4.4. 
Theorem 4.2. Let $M$ be a conformal manifold of dimension $n$ even. Then

$$
\square_{n / 2-2} W_{A_{1} A_{2} B_{1} B_{2}}=K(n) X_{A_{1}} Z_{A_{2}}{ }^{a} X_{B_{1}} Z_{B_{2}}{ }^{b} \mathbb{O}_{a b} \text {. }
$$

Here $K(n)$ is a known nonzero constant depending on $n$, and $\mathscr{O}_{a b} \in \mathscr{E}_{(a b)_{0}}[2-n]$ is the Fefferman-Graham obstruction tensor. It is conformally invariant and natural.

We have $K(4)=-8$. In dimensions at least 6 , the constant $K(n)$ is given by $(n-4) k(n)$, where $k(n)$ is given in (38). Note that $\square_{n / 2-2} W \in \mathscr{T}^{2,2}[2-n]$. The theorem states that its components vanish in all factors of the composition series (11) for $\mathscr{T}^{2,2}[2-n]$, except for the (injecting) factor $\mathscr{E}_{1,1}[-2]=\mathscr{E}^{1,1}[2-n]$, and the term here is the obstruction, up to scale.

Theorem 4.3. The obstruction tensor $\mathrm{O}_{a b}$ vanishes on conformally Einstein manifolds.

Proof. A conformally Einstein manifold $M$ admits a parallel standard tractor $\square$ (see [Gover and Nurowski 2006]) such that $\sigma:=\square^{A} X_{A} \neq 0$ is an Einstein scale. It follows immediately that $\llbracket$ annihilates the tractor curvature $\Omega_{b c}{ }^{D}{ }_{E}$ :

$$
\nabla_{c} \rrbracket^{D}=0 \Longrightarrow \Omega_{b c}{ }^{D}{ }_{E} \rrbracket^{E}=\left[\nabla_{b}, \nabla_{c}\right]^{D}=0 .
$$

Also since $\llbracket$ is parallel, viewing it as a multiplication operator, it is clear that $[D, 0]=0$. From (27) (see also [Čap and Gover 2003]) we have $W_{A_{1} A_{2}} D_{E}=$ $(3 /(n-2)) D^{A_{0}} X_{A_{0}} Z_{A_{1}}{ }^{b} Z_{A_{2}}{ }^{c} \Omega_{b c}{ }^{D}{ }_{E}$. Thus $W_{B C D E} \rrbracket^{E}=0$.

By Theorem 4.1 there is a formula for $\square_{n / 2-2} W_{A_{1} A_{2} B_{1} B_{2}}$ which is polynomial in $\square, D, W, X, h$, and $h^{-1}$, and in this formula each of the indices $A_{1}, A_{2}, B_{1}$, and $B_{2}$ appears on a $W$ tractor. On the other hand, since $\llbracket$ is parallel and of weight 0 , it commutes with the operators in this expression for $\square_{n / 2-2} W_{A_{1} A_{2} B_{1} B_{2}}$. Thus

$$
\square^{B_{1}} \square_{n / 2-2} W_{A_{1} A_{2} B_{1} B_{2}}=0,
$$

since $\llbracket{ }^{A} W_{A B C D}=0$.

From [Gover and Nurowski 2006] we have $\rrbracket^{A}=(1 / n) D^{A} \sigma$. Thus from the expression (16) for the tractor-D operator, we have the expression

$$
\left[0^{A}\right]_{g}=\sigma Y^{A}-(1 / n) J \sigma X^{A}
$$

for $\rrbracket^{A}$ in terms of the (Einstein) metric $g:=\sigma^{-2} g$. (Recall that if $\nabla$ is the LeviCivita connection determined by $g=\sigma^{-2} g$, then tautologically $\nabla \sigma=0$.) In particular, in this scale, we have $\rrbracket^{A} Z_{A}{ }^{a}=0$. Thus from Theorem 4.2 above,

$$
4(K(n))^{-1} Z^{A_{2}}{ }_{a} Z^{B_{2}}{ }_{b} \square^{A_{1}} \rrbracket^{B_{1}} \emptyset_{n / 2-2} W_{A_{1} A_{2} B_{1} B_{2}}=\sigma^{2} \widehat{O}_{a b} .
$$

But from (33), the left-hand side vanishes, and hence $O_{a b}=0$ on $M$. 
Obtaining the obstruction tensor via a conformally invariant operator on a tractor field as in Theorem 4.2 enables us to relate it to other conformally invariant operators associated with the deformation complex, by ideas along the lines of the curved translation principle of Eastwood [1996] and collaborators. This is the idea behind Theorem 2.3, which we are now ready to prove. Related generalisations of the curved translation principle have been explored in depth in the setting of operators on differential forms [Branson and Gover 2005].

Proof of Theorem 2.3. We first construct $\mathbb{B}$ and prove (iii). Let $W^{2,2}$ denote the quotient of $\mathbb{T}^{2,2}[-2]$ by the subbundle which is the kernel of the bundle map $\mathbb{T}^{2,2}[-2] \rightarrow \mathbb{T}^{3} \otimes \mathbb{T}^{3}$ given by

$$
U_{A_{2} A_{3} B_{2} B_{3}} \mapsto X_{A_{1}} X_{B_{1}} U_{A_{2} A_{3} B_{2} B_{3}} .
$$

We write $W_{2,2}$ for the subbundle of $\mathbb{T}^{2,2}[2-n]$ consisting of tractors which are annihilated by any contraction with $X$, and write $\mathscr{W}^{2,2}$ and $\mathscr{W}_{2,2}$ for the section spaces of $W^{2,2}$ and $W_{2,2}$, respectively. Note that complete contractions between elements of $\mathbb{T}^{2,2}[-2]$ and sections of $\mathbb{T}^{2,2}[2-n]$ take values in $E[-n]$. Hence there is a conformally invariant pairing between $\mathscr{T}^{2,2}[-2]$ and $\mathscr{T}^{2,2}[2-n]$. It is clear that the contractions between elements of $\mathbb{T}^{2,2}[-2]$ and sections of $\mathbb{T}^{2,2}[2-n]$ induce a well-defined bundle map

$$
\langle\cdot, \cdot\rangle: W^{2,2} \otimes W_{2,2} \rightarrow E[-n],
$$

and so there is also a conformally invariant pairing between $\mathcal{W}^{2,2}$ and $\mathcal{W}_{2,2}$.

Given a section $U_{A B C D} \in \mathscr{T}^{2,2}[-2]$, let $\left[U_{A B C D}\right]$ be its image in the quotient space $W^{2,2}$. From the tractor composition series (6) (see also (10) and the discussion there), it follows easily that the space $\mathcal{W}^{2,2}$ has a composition series

$$
\mathscr{E}^{1,1}[2] \bullet \mathscr{E}^{2,1}[2] \bullet \mathscr{E}^{2,2}[2]
$$

and that the injection $\mathrm{I}: \mathscr{E}^{2,2}[2] \rightarrow \mathscr{W}^{2,2}$ is given by

$$
u_{a b c d} \mapsto\left[Z_{A}{ }^{a} Z_{B}^{b} Z_{C}^{c} Z_{D}^{d} u_{a b c d}\right] .
$$

The differential operator $\mathbb{D}: \mathscr{W}^{2,2} \rightarrow \mathscr{T}^{2,2}[-2]$ given by

$$
\left[U_{A_{2} A_{3} B_{2} B_{3}}\right] \mapsto \frac{9}{n(n-2)} \mathbb{Y}_{2,2} D^{A_{1}} D^{B_{1}} X_{A_{1}} X_{B_{1}} U_{A_{2} A_{3} B_{2} B_{3}}
$$

is clearly well-defined and conformally invariant. Here $\mathbb{Y}_{2,2}$ is the bundle map executing the projection of $\mathbb{T}^{2}[-1] \otimes \mathbb{T}^{2}[-1]$ onto the direct summand $\mathbb{T}^{2,2}[-2]$. We write $\mathbb{D}^{*}$ for the formal adjoint of $\mathbb{D}$. This is a conformally invariant operator

$$
\mathbb{D}^{*}: \mathscr{T}^{2,2}[2-n] \rightarrow \mathcal{W}_{2,2}
$$


On the other hand, from Theorem 4.1 there is a conformally invariant Laplaciantype operator $\square_{n / 2-2}: \mathscr{T}^{2,2}[-2] \rightarrow \mathscr{T}^{2,2}[2-n]$. Thus we have the composition

$$
\mathbb{D}^{*} \square_{n / 2-2} \mathbb{D}: \mathcal{W}^{2,2} \rightarrow \mathcal{W}_{2,2} \text {. }
$$

The operator $\mathbb{B}$ in the theorem is (up to a constant multiple) simply the composition

$$
\left(\mathbb{D}^{*} \square_{n / 2-2} \mathbb{D I}=: \mathbb{B}\right): \mathscr{\mathscr { C }}^{2,2}[2] \rightarrow \mathcal{W}_{2,2}
$$

By construction this is natural and conformally invariant.

Now in a conformal scale, $(\mathbb{D} \mathrm{I}(u))_{B C E F}$ is given explicitly by

$$
\begin{gathered}
(n-4)\left((n-3) Z_{B}^{b} Z_{C}{ }^{c} Z_{E}^{e} Z_{F}{ }^{f} u_{b c e f}-2 Z_{B}^{b} Z_{C}{ }^{c} X_{[E} Z_{F]}{ }^{f} \nabla^{e} u_{e f b c}\right. \\
\left.-2 X_{[B} Z_{C]}{ }^{c} Z_{E}{ }^{e} Z_{F}{ }^{f} \nabla^{b} u_{b c e f}\right) \\
+4 X_{[B} Z_{C]}^{c} X_{[E} Z_{F]}^{f}\left(\nabla^{(b} \nabla^{e)} u_{b c e f}+(n-3) P^{b e} u_{b c e f}\right) .
\end{gathered}
$$

Thus from (28) and a minor calculation, $\mathbb{D}(\mathrm{I}(C))_{A B C D}=(n-3) W_{A B C D}$, where $C$ is the Weyl curvature. So by Theorem 4.2, we have

$$
\left(\square_{n / 2-2} \mathbb{D I I C}\right)_{A_{2} A_{3} B_{2} B_{3}}=(n-3) K(n) X_{A_{2}} Z_{A_{3}}{ }^{a} X_{B_{2}} Z_{B_{3}}{ }^{b} \mathbb{O}_{a b} \text {. }
$$

That is, $\square_{n / 2-2} \mathbb{D I C}$ takes values in the factor $\mathscr{E}_{1,1}[-2]$ in the composition series for $\mathscr{T}^{2,2}[2-n]$. (Note that this factor is a conformally invariant subspace.) Now the formal adjoint of the tractor-D operator is again the tractor-D operator [Branson and Gover 2001]. So

$$
\mathbb{D}^{*} X_{A_{2}} Z_{A_{3}}{ }^{a} X_{B_{2}} Z_{B_{3}}{ }^{b} O_{a b}=\frac{9}{n(n-2)} X^{B_{1}} X^{A_{1}} D_{B_{1}} D_{A_{1}} X_{A_{2}} Z_{A_{3}}{ }^{a} X_{B_{2}} Z_{B_{3}}{ }^{b} \mathbb{O}_{a b} .
$$

But a short calculation using (9) and (16) shows that this operation just returns $4(n-4)(n-3) X_{A_{2}} Z_{A_{3}}{ }^{a} X_{B_{2}} Z_{B_{3}}{ }^{b} O_{a b}$, and this proves part (iii) of the theorem. All nonvanishing multiples can be absorbed into the definition of $\mathbb{B}$.

We treat now part (i). We need to show that $I^{*} \mathbb{B}$ has order $n-4$ and is nontrivial. Since by construction there is a universal natural expression for the operator $L$, it is sufficient to establish this on the standard conformal sphere. Recall that $\square_{n / 2-2}$ has leading term $\Delta^{n / 2-2}$. Thus $\square_{n / 2-2}$ is elliptic, since the sphere has Riemannian signature. From (34) it is clear that $\mathbb{D I}: \mathscr{E}^{2,2}[2] \rightarrow \mathscr{T}^{2,2}[-2]$ is a differential splitting operator; there is a bundle homomorphism $\mathrm{J}: \mathbb{T}^{2,2}[-2] \rightarrow E^{2,2}[2]$ such that $\mathrm{J} \mathbb{D I}$ is the identity on $\mathscr{E}^{2,2}[2]$. Thus on any manifold, $\mathscr{R}\left(\mathbb{D I}: \mathscr{E}^{2,2}[2] \rightarrow \mathscr{T}^{2,2}[-2]\right)$ is infinite-dimensional, and it follows immediately that $\square_{n / 2-2} \mathbb{D I}$ is nontrivial on the standard conformal sphere. The action of $\square_{n / 2-2} \mathbb{D I}$ on $\mathscr{E}^{2,2}[2]$ takes values in $\mathscr{T}^{2,2}[2-n]$. The composition series for $\mathscr{T}^{2,2}[2-n]$ is given by (11) with $w=2-n$. From this we see, for example, that there is a canonical projection from $\mathscr{T}^{2,2}[2-n]$ to $\mathscr{E}^{1,1}[6-n]=\mathscr{E}_{1,1}[2]$ with which one can compose the operator 
$\square_{n / 2-2} \mathbb{D I}: \mathscr{E}^{2,2}[2] \rightarrow \mathscr{T}^{2,2}[2-n]$. By construction, this is a conformally invariant operator $\mathscr{E}^{2,2}[2] \rightarrow \mathscr{E}_{1,1}[2]$. On the other hand, from the classification of operators on conformally flat structures discussed in Section 2, the only conformally invariant operators on $\mathscr{E}^{2,2}$ [2] taking values in irreducible bundles are as follows: there is an operator $\mathscr{E}^{2,2}[2] \rightarrow \mathscr{E}^{3,2}[2]$ and an operator $\mathscr{E}^{2,2}[2] \rightarrow \mathscr{E}_{2,2}[-2]$. From elementary weight considerations, we know the latter has order $n-4$. Thus the composition described must be trivial. Continuing in this fashion and using (8), one concludes that $\square_{n / 2-2} \mathbb{D I}$ takes values in the subspace $\mathscr{W}_{2,2}=\mathscr{E}_{2,2}[-2] \in \mathscr{E}_{2,1}[-2] \in \mathscr{E}_{1,1}[-2]$, and the composition of $\square_{n / 2-2} \mathbb{D I}$ with projection to $\mathscr{E}_{2,2}[-2]$ is necessarily nontrivial. This composition is thus up to scale the unique conformally invariant operator between these bundles (on the conformal sphere). To finish, note that on the one hand, $I^{*} \mathbb{D}^{*}$ is the formal adjoint of a splitting operator for $\mathscr{E}^{2,2}[2]$ and therefore acts as a multiple of the identity on the component $\mathscr{E}_{2,2}[-2]$. On the other hand, I* $\mathbb{D}^{*}$ must annihilate the components $\mathscr{E}_{2,1}[-2]$ and $\mathscr{E}_{1,1}[-2]$, since these have higher total order than the target bundle $\mathscr{E}_{2,2}[-2]$ for the composition and a natural differential operator cannot lower order.

Finally, we consider (ii). Let us first consider the case of a flat Riemannian or pseudo-Riemannian structure. Thus all curvature will vanish, until we note otherwise. If $F_{a_{1} a_{2}}$ is a 2-form, then $\Delta^{n / 2-2} D^{A_{0}} X_{A_{0}} Z_{A_{1}}{ }^{a_{1}} Z_{A_{2}}{ }^{a_{2}} F_{a_{1} a_{2}}$ is well understood as a special case of [Branson and Gover 2005, Proposition 4.6]. The nonzero components of this have values in a subbundle of $\mathscr{T}^{2}[2-n]$ with composition series $\mathscr{E}_{2} \oplus \mathscr{E}_{1}$. These components are (up to an overall nonzero constant multiple $\left((\delta d)^{n / 2-2} F, a \delta(d \delta)^{n / 2-2} F\right)$, where $d$ is the exterior derivative, $\delta$ its formal adjoint, and $a$ is a nonzero constant. Composing these components on the left with $(\delta d,(1 / a) d)$ yields $(\delta d+d \delta)^{n / 2-1} F=(-1)^{n / 2-1} \Delta^{n / 2-1} F$. Now on flat structures we have the identity

$$
(n-2)(\mathbb{D I}(u))_{A_{1} A_{2} B_{1} B_{2}}=3 D^{A_{0}} X_{A_{0}} Z_{A_{1}}{ }^{a_{1}} Z_{A_{2}}{ }^{a_{2}} U_{a_{1} a_{2} B_{1} B_{2}},
$$

where $u \in \mathscr{E}^{2,2}[2]$ and $U_{a_{1} a_{2} B_{1} B_{2}}$ is the conformally invariant form-tractor given in scale by letting $w$ equal 2 in the formula

$$
U_{a_{1} a_{2} B_{1} B_{2}}=(n+w-5) Z_{B_{1}}{ }^{b_{1}} Z_{B_{2}}{ }^{b_{2}} u_{a_{1} a_{2} b_{1} b_{2}}+2 X_{B_{1}} Z_{B_{2}}{ }^{b_{2}} \nabla^{b_{1}} u_{a_{1} a_{2} b_{2} b_{1}} .
$$

Thus by viewing $U$ as a 2 -form with values in a tractor bundle and replacing $\nabla$, $d$, and $\delta$ with their tractor connection coupled variants in the argument above, we conclude that there is an operator $\overline{\mathbb{A}}$ such that

$$
\overline{\mathbb{A}} \Delta^{n / 2-2} \mathbb{D} \mathrm{I}(u)=\frac{3}{n-2} \overline{\mathbb{A}} \Delta^{n / 2-2} D^{A_{0}} X_{A_{0}} Z_{A_{1}}{ }^{a_{1}} Z_{A_{2}}{ }^{a_{2}} U_{a_{1} a_{2} B_{1} B_{2}}=\Delta^{n / 2-1} U .
$$

We continue with similar considerations, except that now we view $u^{a_{1}} a_{2} b_{1} b_{2}$ as a 2 -form on the $b_{1} b_{2}$ index pair that takes values in $\operatorname{End}(T M)$. If $F$ now indicates a 
2-form of weight $w^{\prime}$, we have

$$
\begin{aligned}
\mathbb{K}(F) & :=\left(3 /\left(n+2 w^{\prime}-2\right)\right) D^{A_{0}} X_{A_{0}} Z_{A_{1}}{ }^{a_{1}} Z_{A_{2}}{ }^{a_{2}} F_{a_{1} a_{2}} \\
& =\left(n+w^{\prime}-4\right) Z_{A_{1}}{ }^{a_{1}} Z_{A_{2}}{ }^{a_{2}} F_{a_{1} a_{2}}+2 X_{A_{1}} Z_{A_{2}}{ }^{a_{2}} \nabla^{a_{1}} F_{a_{2} a_{1}} .
\end{aligned}
$$

So if $w^{\prime}=1$ in particular, then the formula on the right-hand side agrees with (35) with $w=2$. In formally calculating $\Delta^{n / 2-1} U^{a_{1}} a_{2} B_{1} B_{2}$ using the identities (9) and the Leibniz rule to obtain a formula polynomial in $u, \nabla$, the metric $g$, its inverse, and the projectors $X, Y$, and $Z$, we may ignore the $a_{1}$ and $a_{2}$. Their contribution is buried in the meaning of the Levi-Civita connection $\nabla$. Now for a 2-form $F$ of weight 1 , we have that on flat structures, $\Delta^{n / 2-1} \mathbb{K}(F)$ takes values in $\mathscr{E}_{2}[-1] \ominus \mathscr{E}_{1}[-1]$ and has the form $\left(\left((3-n)(\delta d)^{n / 2-1}+(d \delta)^{n / 2-1}\right) F, *\right)$ up to an overall nonzero multiple [Branson and Gover 2005]. Here $*$ indicates some term, the details of which will not concern us. We note that the first entry gives an elliptic operator on $F$; we may act on this with the operator $\delta d+(3-n) d \delta$ to yield $(3-n)(-1)^{n / 2} \Delta^{n / 2} F$. Thus there is a linear differential operator $\overline{\mathbb{A}}_{2}$ such that $\overline{\mathbb{A}}_{2} \Delta^{n / 2-1} U=\Delta^{n / 2} u$.

Combining these observations, we see that there is a linear differential operator $\overline{\mathbb{A}}_{3}$ such that $\overline{\mathbb{A}}_{3} \Delta^{n / 2-2} \mathbb{D} \mathrm{I}(u)=\Delta^{n / 2} u$. Finally, one can easily verify directly that $\mathbb{D}^{*}$ is differentially invertible as a graded differential operator on the subspace $W_{2,2}$. (That is, its inverse is also a graded differential operator. The point is that in terms of a splitting of $W_{2,2}$ determined by a choice of conformal scale, a straightforward calculation shows that $\mathbb{D}^{*}$ takes $(u, v, w)$ to $(k u, \ell v+\delta \cdot u, m w+\delta \cdot v+\delta \cdot \delta \cdot u)$, where $k, \ell$, and $m$ are nonzero integers, $\delta$. indicates a divergence operator, and $\delta \cdot \delta$. a double divergence operator.) Thus with $\overline{\mathbb{B}}$ defined to be the necessary multiple of $\overline{\mathrm{A}}_{3}\left(\mathbb{D}^{*}\right)^{-1}$, we have (ii) for flat structures. But now the result follows in general, since moving to curved structures yields the same formal calculation, except that at each stage the differential operators concerned may have additional lower order terms involving curvature. It is easily checked that these terms can only yield terms of order lower than $n$ in the final calculation of $\overline{\mathbb{B}} \mathbb{B}$.

Proof of Proposition 2.2. We treat $\mathrm{L}$ first. We already have $\mathrm{L}=\mathrm{Bi}{ }^{*} \mathrm{Bi}$ in dimension 6 , and so we shall assume that $n \geq 8$. Let us denote by

$$
\mathbb{U}: \mathscr{E}^{2,2}[2] \rightarrow \mathscr{E}^{2} \otimes \mathscr{T}^{2}
$$

the conformally invariant operator given by (35). We write $d^{\nabla}$ for the tractor connection coupled exterior derivative and $\delta^{\nabla}$ for its formal adjoint. Thus for example for $U \in \mathscr{E}^{2} \otimes \mathscr{T}^{2}$ we have $\left(d^{\nabla} U\right)_{a_{0} a_{1} a_{2} B_{1} B_{2}}=3 \nabla_{a_{0}} U_{a_{1} a_{2} B_{1} B_{2}}$. It is straightforward using (9) to verify that on conformally flat structures, the composition $d^{\nabla} \mathbb{U}$ can be reexpressed in the form $\mathbb{M B i}$, where $\mathbb{M}: \mathscr{E}^{3,2}[2] \rightarrow \mathscr{E}^{3} \otimes \mathscr{T}$ is a conformally invariant first-order differential splitting operator. 
There are conformally invariant formally self-adjoint operators $L_{k}: \mathscr{E}^{k} \rightarrow \mathscr{E}_{k}$, $0 \leq k \leq n$, with leading term $(\delta d)^{k}$. These are the "long operators" for the de Rham complex given in [Branson and Gover 2005]. It is shown there that there are natural linear differential operators $Q_{k+1}$ such that $L_{k}=\delta Q_{k+1} d$.

Now suppose we are on a contractible (conformally flat) manifold. This suffices for our present purposes. Then the tractor bundle is flat and trivial. It follows that there are conformally invariant and formally self-adjoint tractor-coupled variants of the $L_{k}$,

$$
L_{k}^{\nabla}: \mathscr{E}^{k} \otimes \mathscr{T}^{2} \rightarrow \mathscr{E}_{k} \otimes \mathscr{T}^{2} .
$$

These are obtained by starting with the natural formulae for $L_{k}=\delta Q_{k+1} d$ and formally replacing each instance of $d, \delta$, and the Levi-Civita connection with, respectively, $d^{\nabla}, \delta^{\nabla}$, and the Levi-Civita tractor-coupled connection. By construction the result has a factorisation $L_{k}^{\nabla}=\delta^{\nabla} Q_{k+1}^{\nabla} d^{\nabla}$ for some differential operator $Q_{k+1}^{\nabla}$.

Observe that by composition, we have a formally self-adjoint conformally invariant operator $\mathbb{U}^{*} L_{2}^{\nabla} \mathbb{U}: \mathscr{E}^{2,2}[2] \rightarrow \mathscr{E}_{2,2}[-2]$, where $\mathbb{U}^{*}$ is the formal adjoint of $\mathbb{U}$. We will reexpress this. By taking formal adjoints, we have $\mathbb{U}^{*} \delta^{\nabla}=\mathrm{Bi}^{*} \mathbb{M}^{*}$ from $d^{\nabla} \mathbb{U}=\mathbb{M B i}$. Thus we obtain an operator

$$
\mathrm{Bi}^{*} \mathrm{M}^{*} Q_{3}^{\nabla} \mathrm{MBi}: \mathscr{E}^{2,2}[2] \rightarrow \mathscr{E}_{2,2}[-2] .
$$

The result follows from the uniqueness of $L$, provided the displayed operator is nontrivial. It is clearly sufficient to establish this for Riemannian signature structures and at a flat metric within the conformal class. We use the alternative expression $\mathbb{U}^{*} \delta^{\nabla} Q_{3}^{\nabla} d^{\nabla} \mathbb{U}=\mathrm{Bi}^{*} \mathrm{M}^{*} Q_{3}^{\nabla} \mathrm{MBi}$. On flat structures, $Q_{3}=(d \delta)^{n / 2-3}$, and so $Q_{3}^{\nabla}=\left(d^{\nabla} \delta^{\nabla}\right)^{n / 2-3}$. It follows that for $u \in \mathscr{E}^{2,2}$ [2] of compact support, $\delta^{\nabla}\left(d^{\nabla} \delta^{\nabla}\right)^{n / 2-3} d^{\nabla} \mathbb{U}(u)$ vanishes if and only if $d^{\nabla} \cup(u)$ vanishes, since the tractor connection is flat. (Suppose $d^{\nabla} \mathbb{U}(u) \neq 0$. Then there exists a parallel $T \in \mathscr{T}^{2}$ such that $T^{B_{1} B_{2}}\left(d^{\nabla} \cup(u)\right)_{a_{0} a_{1} a_{2} B_{1} B_{2}} \neq 0$. In other words, if $f_{a_{1} a_{2}}:=T^{B_{1} B_{2}} \cup(u)_{a_{1} a_{2} B_{1} B_{2}}$, then $d f \neq 0$. But on the other hand, if $0=T^{B_{1} B_{2}}\left(\delta^{\nabla}\left(d^{\nabla} \delta^{\nabla}\right)^{n / 2-3} d^{\nabla} \cup(u)\right)_{a_{0} a_{1} a_{2} B_{1} B_{2}}$, then $(\delta d)^{n / 2-2} f=0$ which implies $d f=0$.) This is equivalent to $\operatorname{MBi}(u)$ vanishing. Since $\mathbb{M}$ is a differential splitting operator, this in turn is equivalent to $\operatorname{Bi}(u)=0$. Thus the composition $\delta^{\nabla} Q_{3}^{\nabla} d^{\nabla \mathbb{U}: \mathscr{E}^{2,2}}[2] \rightarrow \mathscr{E}_{2} \otimes \mathscr{T}^{2}$ is nontrivial. Now it is easily verified that $\mathscr{E}_{2,2}[-2]$ turns up with multiplicity 1 in the composition series for $\mathscr{E}_{2} \otimes \mathscr{T}^{2}$. It follows by an exact analogue of the argument used on page 331 that $\delta^{\nabla} Q_{3}^{\nabla} d^{\nabla} \mathbb{U}$ only takes values in $\mathscr{E}_{2,2}[-2]$ and composition factors of higher total order. Thus on the range of this operator, $\mathbb{U}^{*}$ acts as a nonzero multiple of the projection to the component $\mathscr{E}_{2,2}[-2]$. (Recall that $\mathbb{U}^{*}$ is the formal adjoint of a differential splitting operator $\mathbb{U}: \mathscr{E}^{2,2}[2] \rightarrow \mathscr{E}^{2} \otimes \mathscr{T}^{2}$, and so it must act as a nonzero multiple of the identity on the component $\mathscr{E}_{2,2}[-2]$. On the other hand, it is differential, so it cannot lower total order.) 
Now we consider the situation for B. We require a conformally invariant differential splitting operator $\Gamma: \mathscr{E}^{1,1}[2] \rightarrow \mathscr{E}^{1} \otimes \mathscr{T}^{2}$ that will in this case play a role analogous to $\mathbb{U}$ above. This is easily constructed explicitly and directly, and can be obtained from a composition of the related operators in Section 5.1 of [Branson and Gover 2002], so we omit the details. Since $\Gamma$ has values in a weight zero adjoint tractor-valued bundle of 1 -forms it is clear that the composition $d^{\nabla} \Gamma$ is conformally invariant. This is easily verified nontrivial. On the other hand, in terms of a metric $g$, the tractor curvature is given by

$$
Z_{B_{1}}{ }^{b_{1}} Z_{B_{2}}{ }^{b_{2}} C_{a_{1} a_{2} b_{1} b_{2}}+\frac{2}{n-3} X_{B_{1}} Z_{B_{2}}{ }^{b_{2}} \nabla^{b_{1}} C_{a_{1} a_{2} b_{2} b_{1}} .
$$

Thus the linearisation, at a conformally flat metric $g_{0}$, of the tractor curvature is $(1 /(n-3)) \mathbb{U C}$. This is manifestly nontrivial, and so via arguments used several times already concerning the uniqueness of irreducible conformally invariant operators, it is straightforward to verify that this operator must agree with $d^{\nabla} \Gamma$ (on conformally flat structures), at least up to scale. We set the scale of $\Gamma$ so that $d^{\nabla} \Gamma=(1 /(n-3)) \cup C$. On flat manifolds, $Q_{2}=(d \delta)^{n / 2-2}$, and so by almost the same argument as for $\mathrm{L}$, we conclude that on conformally flat manifolds, the formally self-adjoint conformally invariant operator $\mathrm{C}^{*} \mathbb{U}^{*} Q_{2}^{\nabla \mathbb{U} \mathrm{C}}$ is nontrivial.

The next theorem shows that for $n$ even, if the ambient curvature is formally Ricci-flat to $O\left(Q^{n / 2-1}\right)$, then a tensor part of the coefficient of $Q^{n / 2-1}$ is a natural conformal invariant of the underlying manifold and so is an obstruction to finding an ambient metric which is Ricci-flat to higher order. For our purposes, the main point is that this is achieved by Theorem 4.4(iii), which recovers this obstruction via a tangential operator acting on the ambient curvature.

Theorem 4.4. For a conformal manifold $M$ of even dimension $n$, let $\boldsymbol{h}$ be an associated ambient metric satisfying $\operatorname{Ric}(\boldsymbol{h})=Q^{n / 2-1} \boldsymbol{B}$. Then we have

(i) $\left.\boldsymbol{B}\right|_{2}$ is equivalent to a tractor $B_{A B} \in \mathscr{E}_{(A B)_{0}}[-n]$ such that $X^{A} B_{A B}=0$.

(ii) The weighted tensor $Z^{A}{ }_{a} Z^{B}{ }_{b} B_{A B}=: \mathscr{O}_{a b}$ is a section of $\mathscr{E}_{(a b)_{0}}[2-n]$.

(iii) For $n \geq 6$, we have

$$
\boldsymbol{\Delta}^{n / 2-2} \boldsymbol{R}_{A_{1} A_{2} B_{1} B_{2}}=k(n) \boldsymbol{X}_{A_{1}} \boldsymbol{X}_{B_{1}} \boldsymbol{B}_{A_{2} B_{2}}+O(Q),
$$

where $k(n)$ is the dimension dependent nonzero constant given above. In dimension 4,

$$
3 \boldsymbol{D}^{A_{0}} \boldsymbol{X}_{A_{0}} \boldsymbol{R}_{A_{1} A_{2} B_{1} B_{2}}=16 \boldsymbol{X}_{A_{2}} \boldsymbol{X}_{B_{1}} \boldsymbol{B}_{A_{1} B_{2}}+O(Q) .
$$

(iv) The tensor $\mathrm{O}_{a b}$ is divergence-free.

(v) The weighted tensor $\mathrm{O}_{a b}$ is a nontrivial natural conformal invariant of the form $\Delta^{n / 2-2} \nabla^{c} \nabla^{d} C_{c a d b}+$ lots $=(n-3) \Delta^{n / 2-2}\left(\Delta P_{a b}-\nabla_{a} \nabla_{b} J\right)+$ lots $(u p$ 
to a constant multiple), and so is an obstruction to finding an ambient metric which is Ricci-flat modulo $O\left(Q^{n / 2}\right)$.

Remarks. 1. The statement of the theorem up to the definition of $\mathrm{O}_{a b}$ in (ii) is a characterisation of the Fefferman-Graham obstruction tensor (Graham, private communication; see also [Fefferman and Graham, in progress]). This gives a complete obstruction to the ambient metric in the sense that if this vanishes, then the ambient construction may be continued to all orders [Fefferman and Graham 1985]. Hence $\mathrm{O}_{a b}$ is the usual obstruction tensor, as claimed in Theorem 4.2. Thus part (iii), above, gives a new ambient formula for the Fefferman-Graham obstruction tensor.

2. From (25) it follows easily that $\mathrm{O}_{a b}$ may be equally viewed as an obstruction to obtaining an ambient metric which is harmonic for $\boldsymbol{\Delta}$ in the sense that $\boldsymbol{\Delta} \boldsymbol{R}$ vanishes to all orders. See also the remark on page 325 .

3. It should be pointed out that

$$
\boldsymbol{\Delta}^{n / 2-3} \boldsymbol{\Delta} \boldsymbol{R}_{A_{1} A_{2} B_{1} B_{2}}=k(n) \boldsymbol{X}_{A_{1}} \boldsymbol{X}_{B_{1}} \boldsymbol{B}_{A_{2} B_{2}}+O(Q)
$$

is an alternative ambient formula for the obstruction, and we could replace the $\boldsymbol{\Delta}^{n / 2-3}$ by $\boldsymbol{\Delta}_{\alpha}^{n / 2-3}$ in this formula.

Proof of Theorems 4.2 and 4.4. As above, we write $\operatorname{Ric}$ for $\operatorname{Ric}(\boldsymbol{h})$. It is immediate that $\boldsymbol{B}$ is symmetric and homogeneous of weight $-n$. Also from (5) it follows that $\boldsymbol{X}^{A} \boldsymbol{B}_{A B}=0$. So $\left.\boldsymbol{B}\right|_{2}$ is equivalent to a tractor field $B_{A B} \in \mathscr{T}_{(A B)}[-n]$ satisfying $X^{A} B_{A B}=0$. From this last equality and (8), it is clear that $\mathrm{O}_{a b}$ is conformally invariant, while from the weight and symmetry of $B_{A B}$, it follows that $\mathcal{O}_{a b} \in \mathscr{E}_{(a b)}[2-n]$. For parts (i) and (ii), it remains to show that both $B_{A B}$ and $\mathrm{O}_{a b}$ are trace-free.

First we consider the case $n \neq 4$. Note that since $\nabla_{A} Q=2 X_{A}$, we have

$$
\nabla_{A_{1}} \nabla_{B_{1}} \mathbf{R i c}_{A_{2} B_{2}}=(n-2)(n-4) Q^{n / 2-3} \boldsymbol{X}_{A_{1}} \boldsymbol{X}_{B_{1}} \boldsymbol{B}_{A_{2} B_{2}}+O\left(Q^{n / 2-2}\right) .
$$

From (21) and (25) together with a short computation, it follows that

$$
\boldsymbol{\Delta}^{n / 2-2} \boldsymbol{R}_{A_{1} A_{2} B_{1} B_{2}}=k(n) \boldsymbol{X}_{A_{1}} \boldsymbol{X}_{B_{1}} \boldsymbol{B}_{A_{2} B_{2}}+O(Q),
$$

as claimed in (iii), where

$$
k(n)=(n-2)(n-4)(-1)^{n / 2-3} 2^{n-4}((n / 2-3) !)^{2} .
$$

(Note that (21) and (25) also give the alternative formula in Remark 3, above.)

Since $\left.(n-4) \boldsymbol{R}\right|_{2}$ is equivalent to the tractor field $W$, it follows from Proposition 4.8 that $\left.(n-4) \boldsymbol{4}^{n / 2-2} \boldsymbol{R}\right|_{2}$ descends to the natural tractor field $\square_{n / 2-2} W$. On the 
other hand, using $\delta_{B}{ }^{A}=X^{A} Y_{B}+Y^{A} X_{B}+Z^{A}{ }_{a} Z_{B}{ }^{b} \delta_{b}{ }^{a}$ and the fact that $X^{A} B_{A B}=0$, we see that

$$
X_{A_{1}} X_{B_{1}} B_{A_{2} B_{2}}=X_{A_{1}} Z_{A_{2}}{ }^{a} X_{B_{1}} Z_{B_{2}}{ }^{b} O_{a b} .
$$

Therefore, $\left.\boldsymbol{X}_{A_{1}} \boldsymbol{X}_{B_{1}} \boldsymbol{B}_{A_{2} B_{2}}\right|_{2}$ is equivalent to the tractor field $X_{A_{1}} Z_{A_{2}}{ }^{a} X_{B_{1}} Z_{B_{2}}{ }^{b} O_{a b}$. This establishes (32) of Theorem 4.2.

Since the left-hand side of (32) is natural, it follows that $X_{A_{1}} X_{B_{1}} B_{A_{2} B_{2}}$ is natural. Hence $O_{a b}=Z^{A_{2}}{ }_{a} Z^{B_{2}}{ }_{b} B_{A_{2} B_{2}}=4 Y^{A_{1}} Y^{B_{1}} Z^{A_{2}}{ }_{a} Z^{B_{2}}{ }_{b} X_{A_{1}} X_{B_{1}} B_{A_{2} B_{2}}$ is likewise natural, as claimed in (v) and Theorem 4.2.

Next we show that $B_{A B}$ and $O_{a b}$ are trace-free. According to Theorem 4.1, the operators $\square_{m}$ preserve tensor type. Since $W_{A_{1} A_{2} B_{1} B_{2}}$ is trace-free, it follows that $\square_{n / 2-2} W_{A_{1} A_{2} B_{1} B_{2}}$ is completely trace-free. Thus $h^{A_{1} B_{2}} X_{A_{1}} X_{B_{1}} B_{B_{2} A_{2}}=0$, by (32) and (39). Since $B_{C D}$ is symmetric and $X^{A} B_{A B}=0$, it follows that $h^{A B} B_{A B}=0$ as claimed. Now using (7) and again that $X^{A} B_{A B}=0$, we see that $\boldsymbol{g}^{a b} \mathcal{O}_{a b}=0$.

We must obtain the corresponding results in dimension 4 . First observe that in any dimension,

$$
3 \boldsymbol{D}^{A_{0}} \boldsymbol{X}_{A_{0}} \boldsymbol{R}_{A_{1} A_{2} B_{1} B_{2}}=(n-2)\left((n-4) \boldsymbol{R}_{A_{1} A_{2} B_{1} B_{2}}+2 \boldsymbol{X}_{A_{1}} \nabla^{C} \boldsymbol{R}_{A_{2} C B_{1} B_{2}}\right)+O(Q),
$$

by (15) and (18). From the contracted Bianchi identity, we have for $n=4$,

$$
3 \boldsymbol{D}^{A_{0}} \boldsymbol{X}_{A_{0}} \boldsymbol{R}_{A_{1} A_{2} B_{1} B_{2}}=8 \boldsymbol{X}_{A_{2}} \nabla_{B_{1}} \mathbf{R i c}_{A_{1} B_{2}}+O(Q)=16 \boldsymbol{X}_{A_{2}} \boldsymbol{X}_{B_{1}} \boldsymbol{B}_{A_{1} B_{2}}+O(Q) .
$$

Relating $W$ to the left-hand side via (27), we conclude that in dimension 4,

$$
W_{A_{1} A_{2} B_{1} B_{2}}=-8 X_{A_{1}} X_{B_{1}} B_{A_{2} B_{2}} .
$$

Comparing this with (28), we have $-2 X_{A_{1}} X_{B_{1}} B_{A_{2} B_{2}}=X_{A_{1}} Z_{A_{2}}{ }^{a} X_{B_{1}} Z_{B_{2}}{ }^{b} B_{a b}$. Thus $\mathrm{O}_{a b}$ is a scalar multiple of the Bach tensor, $\mathrm{O}_{a b}=-\frac{1}{2} B_{a b}$, which is natural and trace-free, by (1). Also, since $W$ is trace-free and $X^{A} B_{A B}=0$, it follows that $B_{A B}$ is trace-free.

It is well known (and easily verified) that the Bach tensor in dimension 4 is divergence-free. For (iv) we need the analogous result in other dimensions. First note that a short calculation using the formula (16) for the tractor-D operator and the identities (9) for the connection shows that

$$
2 D^{A_{1}} X_{A_{1}} Z_{A_{2}}{ }^{a} X_{B_{1}} Z_{B_{2}}{ }^{b} \mathcal{O}_{a b}=(n-4) X_{A_{2}} X_{B_{1}} Z_{B_{2}}{ }^{b} \nabla^{a} \mathscr{O}_{a b} .
$$

So in dimensions other than 4 , it follows that $D^{A_{1}} X_{A_{1}} X_{B_{1}} B_{A_{2} B_{2}}$ and equivalently $\left.\left(\boldsymbol{D}^{A_{1}} \boldsymbol{X}_{A_{1}} \boldsymbol{X}_{B_{1}} \boldsymbol{B}_{A_{2} B_{2}}\right)\right|_{2}$, vanish if and only if $\nabla^{a} \mathscr{O}_{a b}=0$. On the ambient manifold, by (36), $\boldsymbol{D}^{A_{1}} \boldsymbol{X}_{A_{1}} \boldsymbol{X}_{B_{1}} \boldsymbol{B}_{A_{2} B_{2}}$ is

$$
\boldsymbol{D}^{A_{1}} \boldsymbol{\Delta}^{n / 2-3} \boldsymbol{\Delta} \boldsymbol{R}_{A_{1} A_{2} B_{1} B_{2}}+O(Q),
$$


up to a nonzero multiple, since $\boldsymbol{D}$ acts tangentially. We ignore terms $O(Q)$ for much of the remainder of this calculation. The preceding display expands to

$$
(4-n) \nabla^{A_{1}} \boldsymbol{\Delta}^{n / 2-3} \boldsymbol{\Delta} \boldsymbol{R}_{A_{1} A_{2} B_{1} B_{2}}-\boldsymbol{\Delta} \boldsymbol{X}^{A_{1}} \boldsymbol{\Delta}^{n / 2-3} \boldsymbol{\Delta} \boldsymbol{R}_{A_{1} A_{2} B_{1} B_{2}} .
$$

From (5) and (24) we obtain

$$
\begin{aligned}
(4-n)\left[\nabla^{A_{1}}, \boldsymbol{\Delta}\right] & \boldsymbol{\Delta}^{n / 2-4} \boldsymbol{\Delta} \boldsymbol{R}_{A_{1} A_{2} B_{1} B_{2}} \\
& +(6-n) \boldsymbol{\Delta}\left[\nabla^{A_{1}}, \boldsymbol{\Delta}\right] \boldsymbol{\Delta}^{n / 2-5} \boldsymbol{\Delta} \boldsymbol{R}_{A_{1} A_{2} B_{1} B_{2}} \\
& +\cdots-4 \boldsymbol{\Delta}^{n / 2-4}\left[\nabla^{A_{1}}, \boldsymbol{\Delta}\right] \boldsymbol{\Delta} \boldsymbol{R}_{A_{1} A_{2} B_{1} B_{2}} \\
& -2 \boldsymbol{\Delta}^{n / 2-3}\left(\left[\nabla^{A_{1}}, \boldsymbol{\Delta}\right] \boldsymbol{R}_{A_{1} A_{2} B_{1} B_{2}}+\frac{1}{2} \nabla^{A_{1}}\left(\boldsymbol{R} \sharp \sharp \boldsymbol{R}_{A_{1} A_{2} B_{1} B_{2}}\right)\right),
\end{aligned}
$$

after some reorganisation. It remains only to observe that all the terms in this sum are $O(Q)$. First we note that from (21) and (37), it is clear that

$$
\boldsymbol{\Delta} \boldsymbol{R}_{A_{1} A_{2} B_{1} B_{2}}=K Q^{n / 2-3} \boldsymbol{X}_{A_{1}} \boldsymbol{X}_{B_{1}} \boldsymbol{B}_{A_{2} B_{2}}+O\left(Q^{n / 2-2}\right),
$$

for some constant $K$. Thus by (25), each term $\boldsymbol{\Delta}^{k}\left[\nabla^{A_{1}}, \boldsymbol{\Delta}\right] \boldsymbol{\Delta}^{\ell} \boldsymbol{\Delta} \boldsymbol{R}_{A_{1} A_{2} B_{1} B_{2}}$, for $k+\ell=n / 2-4$, is some number times

$$
\boldsymbol{\Delta}^{k}\left[\nabla^{A_{1}}, \boldsymbol{\Delta}\right] Q^{n / 2-3-\ell} \boldsymbol{X}_{A_{1}} \boldsymbol{X}_{B_{1}} \boldsymbol{B}_{A_{2} B_{2}}+O(Q),
$$

since $\left[\nabla^{A_{1}}, \boldsymbol{\Delta}\right]$ is a first-order operator. Now consider the identity obtained from (26) by including the $O\left(Q^{n / 2-2}\right)$ terms which were omitted. From this identity, (5), and the fact that $\nabla Q=2 \boldsymbol{X}$, it follows that $\left[\left[\nabla^{A_{1}}, \Delta\right], Q\right]=0$ identically on the ambient manifold. Thus (41) is $O(Q)$.

Now consider the last term in (40). By direct calculation, we have

$$
\left[\nabla^{A_{1}}, \boldsymbol{\Delta}\right] \boldsymbol{R}_{A_{1} A_{2} B_{1} B_{2}}=-\frac{1}{2} \nabla^{A_{1}}\left(\boldsymbol{R} \sharp \sharp \boldsymbol{R}_{A_{1} A_{2} B_{1} B_{2}}\right)+O\left(Q^{n / 2-2}\right),
$$

and so as required,

$$
\boldsymbol{\Delta}^{n / 2-3}\left(\left[\nabla^{A_{1}}, \boldsymbol{\Delta}\right] \boldsymbol{R}_{A_{1} A_{2} B_{1} B_{2}}+\frac{1}{2} \nabla^{A_{1}}\left(\boldsymbol{R} \sharp \sharp \boldsymbol{R}_{A_{1} A_{2} B_{1} B_{2}}\right)\right)=O(Q) .
$$

Finally, we must show that in general $\mathrm{O}_{a b}$ is nontrivial. Up to scale, $\mathrm{O}_{a b}$ is given by $4 Y^{A_{1}} Y^{B_{1}} Z^{A_{2}}{ }_{a} Z^{B_{2}}{ }_{b} \square_{n / 2-2} W_{A_{1} A_{2} B_{1} B_{2}}$. From (28) and (31), it is clear that $4 Y^{A_{1}} Y^{B_{1}} Z^{A_{2}}{ }_{a} Z^{B_{2}}{ }_{b} W_{A_{1} A_{2} B_{1} B_{2}}$ is at leading order a nonzero multiple of $\nabla^{d} \nabla^{c} C_{c a d b}$. Using that $\square_{n / 2-2}$ has leading term $\Delta^{n / 2-2}$ and (9) to verify that the commutator of $\Delta^{n / 2-2}$ with $4 Y^{A_{1}} Y^{B_{1}} Z^{A_{2}}{ }_{a} Z^{B_{2}}{ }_{b}$ generates only lower order terms, we conclude that $O_{a b}=\ell(n) \Delta^{n / 2-2} \nabla^{d} \nabla^{c} C_{c a d b}+$ lots, where $\ell(n)$ is a nonzero constant. Given the form of the leading term, an elementary exercise shows that this natural tensor cannot vanish in general. 
4A. Conformal Laplacian operators on tractor fields. It remains to prove Theorem 4.1. Our strategy is to first define the operators $\square_{m}^{\alpha}$, which we do via powers of the ambient Laplacian $\boldsymbol{\Delta}_{\alpha}$ in Proposition 4.8, and then rewrite each such power as a combination of compositions of low order tangential operators, each of which has an immediate interpretation as an operator on a tractor bundle. This leads to a simple algorithm for rewriting any operator of this form in terms of basic tractor operators using only the existence of an ambient metric. Two of the key tools are Theorem 4.7, which explains how ambient derivatives of the ambient curvature can be reexpressed in terms of low order tangential operators, and Proposition 4.10, which describes harmonic extensions of tensor fields along 2.

Almost all of the subsequent discussion concerns the ambient manifold $\tilde{M}$ with metric as discussed in Section 3. Occasionally we pause to interpret results on the underlying conformal manifold $M$.

In the generic $n$-even case, some identities, such as (22) and (26), hold to only finite order in $Q$. In many proofs, we will apply the operators $\nabla$ and $\Delta$ to both sides of an identity, and this will reduce the order to which the identity holds. Thus we must keep track of the number of times that we apply $\nabla$ and $\Delta$. In odd dimensions and in the conformally flat case, this is unnecessary since the identities hold to all orders. For simplicity, many of the proofs that follow explicitly treat only the generic $n$-even case. The proofs in the other cases are essentially the same, except that they do not require the operator counts. In addition, we have stated some of the results themselves in the generic $n$-even case only. All results hold as stated. Propositions 4.5 and 4.6, Theorem 4.7, and Lemma 4.11 also hold in general; they hold to all orders in both the odd-dimensional case and the conformally flat case, where the upper bounds stated in the hypotheses of the results no longer apply.

We will often use abbreviated notations. We may abbreviate (26) by writing $[\boldsymbol{\Delta}, \nabla] V=\sum \boldsymbol{R} \nabla V$. It is easily verified that (26) generalises to

$$
\left[\boldsymbol{\Delta}_{\alpha}, \nabla\right] V=\sum \boldsymbol{R} \nabla V+\alpha \sum(\nabla \boldsymbol{R}) V,
$$

which also holds modulo $O\left(Q^{n / 2-2}\right)$ in even dimensions and to infinite order in odd dimensions. For example, let $V$ be any symmetric ambient 2-tensor. In this case (42) stands for

$$
\begin{aligned}
{\left[\boldsymbol{\Delta}_{\alpha}, \nabla_{A}\right] V_{B C}=2(\alpha-1) \boldsymbol{R}_{A}{ }^{P}{ }_{B}{ }^{Q} \nabla_{P} V_{Q C} } \\
+2(\alpha-1) \boldsymbol{R}_{A}{ }^{P}{ }_{C}{ }^{Q} \nabla_{P} V_{B Q}-2 \alpha\left(\nabla_{A} \boldsymbol{R}_{B}{ }^{P}{ }_{C}{ }^{Q}\right) V_{P Q},
\end{aligned}
$$

which holds to the appropriate order. If the $V$ on the left-hand side of (42) has any free indices, then in every term of the right-hand side of (42), each such index either remains attached to $V$ in its original position or moves onto an $\boldsymbol{R}$. Some of the proofs in Section 4 will use this fact, which follows immediately from (26) 
and the definition of $\boldsymbol{R} \sharp \sharp$. The expressions we treat will often involve iterations of operators. To indicate how many operators we are composing in such an iteration, we will use exponents. For example, we might indicate $\nabla_{A} \nabla_{B} \boldsymbol{R}_{C D E F}$ by writing $\nabla^{2} \boldsymbol{R}$. We will often use the symbol $\mathscr{P}$ to denote a partial contraction polynomial. The same $\mathscr{P}$ may denote different polynomials in different parts of a discussion.

We often use the identities (13) and $\nabla Q=2 X$ without explicit mention.

The proof of Theorem 4.1 begins with the development of a useful ambient calculus. This involves a sequence of results.

Proposition 4.5. Suppose that $n$ is even and $M$ is generic. Let an integer $\ell$ be given, and suppose that $0 \leq \ell \leq n / 2-4$. Then on the ambient manifold,

$$
\boldsymbol{\Delta} \nabla^{\ell} \boldsymbol{R}=\sum\left(\nabla^{p} \boldsymbol{R}\right)\left(\nabla^{q} \boldsymbol{R}\right)+O\left(Q^{n / 2-3-\ell}\right),
$$

where $p+q=\ell$. If the $\boldsymbol{R}$ on the left-hand side of (43) has any free indices, then for every term in the summation, these indices appear on an $\boldsymbol{R}$ (as opposed to $a \boldsymbol{\nabla}$ ).

Proof. We use induction. The case $\ell=0$ follows from (22). Suppose next that $0 \leq m \leq n / 2-5$ and that the result holds for $\ell=m$. From this assumption and (26), we have

$$
\begin{aligned}
\boldsymbol{\Delta} \nabla^{m+1} \boldsymbol{R} & =\nabla \boldsymbol{\Delta} \nabla^{m} \boldsymbol{R}+\sum \boldsymbol{R}\left(\nabla^{m+1} \boldsymbol{R}\right)+O\left(Q^{n / 2-2}\right) \\
& =\nabla\left(\sum\left(\nabla^{p} \boldsymbol{R}\right)\left(\nabla^{q} \boldsymbol{R}\right)+O\left(Q^{n / 2-3-m}\right)\right)+\sum \boldsymbol{R}\left(\nabla^{m+1} \boldsymbol{R}\right)+O\left(Q^{n / 2-2}\right) \\
& =\sum\left(\nabla^{s} \boldsymbol{R}\right)\left(\nabla^{t} \boldsymbol{R}\right)+O\left(Q^{n / 2-3-(m+1)}\right) .
\end{aligned}
$$

Here $p+q=m$ and $s+t=m+1$. The use of the inductive assumption and (26) never moves a free index from an $\boldsymbol{R}$ onto a $\boldsymbol{\nabla}$.

Proposition 4.6. Suppose that $n$ is even and $M$ is generic. Let an integer $\ell$ be given, and suppose that $0 \leq \ell \leq n / 2-3$. Then

$$
\boldsymbol{\Delta}^{\ell} \boldsymbol{R}=\sum\left(\nabla^{v_{1}} \boldsymbol{R}\right) \cdots\left(\nabla^{v_{j}} \boldsymbol{R}\right)+O\left(Q^{n / 2-2-\ell}\right) .
$$

The number of factors in a term may vary from term to term, but in any case, $v_{i} \leq \ell$ for $1 \leq i \leq j$. If $A, B, C$, and $D$ denote the indices of the $\boldsymbol{R}$ on the left-hand side, then for each term in the sum, these indices are on an $\boldsymbol{R}$.

Proof. We again use induction. Suppose that $0 \leq m \leq n / 2-4$ and that the result holds for $\ell=m$. Then

$$
\boldsymbol{\Delta}^{m+1} \boldsymbol{R}=\boldsymbol{\Delta}\left(\sum\left(\nabla^{v_{1}} \boldsymbol{R}\right) \cdots\left(\nabla^{v_{j}} \boldsymbol{R}\right)+O\left(Q^{n / 2-2-m}\right)\right) .
$$

By expanding the right-hand side above using the Leibniz rule and the formula $\boldsymbol{\Delta}=\nabla^{A} \nabla_{A}$, we obtain an expression $\sum\left(\nabla^{u_{1}} \boldsymbol{R}\right) \cdots\left(\nabla^{u_{k}} \boldsymbol{R}\right)+O\left(Q^{n / 2-2-(m+1)}\right)$ plus a sum of the form $\sum\left(\Delta \nabla^{t_{0}} \boldsymbol{R}\right)\left(\nabla^{t_{1}} \boldsymbol{R}\right) \cdots\left(\nabla^{t_{s}} \boldsymbol{R}\right)$. In each case, $u_{i} \leq m+1$ and $t_{i} \leq m$. But by Proposition $4.5, \Delta \nabla^{t_{0}} \boldsymbol{R}=\sum\left(\nabla^{p} \boldsymbol{R}\right)\left(\nabla^{q} \boldsymbol{R}\right)+O\left(Q^{n / 2-3-t_{0}}\right)$, 
where $p+q=t_{0} \leq m$. Thus $\boldsymbol{\Delta} \nabla^{t_{0}} \boldsymbol{R}=\sum\left(\nabla^{p} \boldsymbol{R}\right)\left(\nabla^{q} \boldsymbol{R}\right)+O\left(Q^{n / 2-2-(m+1)}\right)$. The use of the inductive assumption and Proposition 4.5 never moves an index from an $R$ onto a $\nabla$.

Theorem 4.7. Suppose that $n$ is even and $M$ is generic. Let $\boldsymbol{h}$ be an ambient metric for a conformal manifold of dimension $n$. Given $t \geq 0$ and $u \geq 0$, suppose that $t+u \leq n / 2-3$. Then there is a partial contraction $\mathscr{P}$, polynomial in $\boldsymbol{D}_{A}$, $\boldsymbol{R}_{A B C D}, \boldsymbol{X}_{A}, \boldsymbol{h}_{A B}$, and its inverse $\boldsymbol{h}^{A B}$, such that

$$
\nabla^{t} \boldsymbol{\Delta}^{u} \boldsymbol{R}=\mathscr{P}+O\left(Q^{n / 2-2-t-u}\right) .
$$

Each term of $\mathscr{P}$ is of degree at least 1 in $\boldsymbol{R}_{A B C D}$. If , in (45), $\boldsymbol{R}$ has any free indices, then in $\mathscr{P}$ these indices always appear on an $\boldsymbol{R}$.

Proof. By Proposition 4.6, we may write

$$
\nabla^{t} \boldsymbol{\Delta}^{u} \boldsymbol{R}=\sum\left(\nabla^{v_{1}} \boldsymbol{R}\right) \cdots\left(\nabla^{v_{j}} \boldsymbol{R}\right)+O\left(Q^{n / 2-2-u-t}\right),
$$

where $v_{i} \leq t+u$ for each $i$. If the $\boldsymbol{R}$ on the left-hand side has any free indices, then for each term in the sum, these indices always appear on an $\boldsymbol{R}$; this follows from Proposition 4.6. To complete the proof, we show that if $0 \leq \ell \leq n / 2-3$, then $\nabla^{\ell} \boldsymbol{R}=\mathscr{P}+O\left(Q^{n / 2-2-\ell}\right)$. We use induction. Suppose that $1 \leq m \leq n / 2-3$, and suppose that $\nabla^{\ell} \boldsymbol{R}=\mathscr{P}+O\left(Q^{n / 2-2-\ell}\right)$ whenever $0 \leq \ell \leq m-1$. By (14) we have

$$
\boldsymbol{D}_{A} \boldsymbol{\nabla}^{m-1} \boldsymbol{R}=(n-2 m-4) \boldsymbol{\nabla}_{A} \boldsymbol{\nabla}^{m-1} \boldsymbol{R}-\boldsymbol{X}_{A} \boldsymbol{\Delta} \boldsymbol{\nabla}^{m-1} \boldsymbol{R} .
$$

Note that $n-2 m-4>0$. Also observe that each $\boldsymbol{R}$ has the same indices. From this equation and Proposition 4.5, we conclude that

$$
\nabla^{m} \boldsymbol{R}=\boldsymbol{D}_{A} \nabla^{m-1} \boldsymbol{R}+\boldsymbol{X}_{A}\left(\sum\left(\nabla^{p} \boldsymbol{R}\right)\left(\nabla^{q} \boldsymbol{R}\right)+O\left(Q^{n / 2-3-(m-1)}\right)\right),
$$

where $p+q \leq m-1$. Also note that if $\boldsymbol{R}$ on the left-hand side of this equation has any free indices, then in each term of the right-hand side, these indices always appear on an $\boldsymbol{R}$. We now see that $\nabla^{m} \boldsymbol{R}=\mathscr{P}+O\left(Q^{n / 2-2-m}\right)$, from our inductive assumption.

Remark. Theorem 4.7 shows that when $n \neq 4$, an ambient partial contraction $\left.\nabla^{t} \boldsymbol{\Delta}^{u} \boldsymbol{R}\right|_{2}$ is equivalent to a conformal invariant which is obtained by taking a partial contraction polynomial in $D, W, X, h$, and its inverse $h^{-1}$. Moreover in each case, via the inductive steps of the proof, one obtains the explicit formula for the invariant as a partial contraction of these quantities. More generally, this shows that any "Weyl invariant" [Bailey et al. 1994b; Fefferman 1979] arising from a complete (partial) contraction of ambient tensors of the form (45) is contained in the space of invariants generated by complete (partial) contractions of the expressions polynomial in the tractor operators and fields $D, W, X, h$, and $h^{-1}$. Furthermore, there is an explicit algorithm for finding the tractor formula, given the formula for 
the ambient invariant. This is a slight generalisation of a result along these lines obtained in [Čap and Gover 2003].

The next proposition is a simple generalisation of results in [Branson and Gover 2005; Graham et al. 1992].

Proposition 4.8. For every integer $m \geq 1$ and every ambient homogeneous tensor space $\mathcal{T}^{\Phi}(m-n / 2)$,

$$
\boldsymbol{\Delta}_{\alpha}^{m}: \mathcal{T}^{\Phi}(m-n / 2) \rightarrow \mathcal{T}^{\Phi}(-m-n / 2)
$$

is tangential and so determines a conformally invariant operator

$$
\square_{m}^{\alpha}: \mathscr{T}^{\Phi}[m-n / 2] \rightarrow \mathscr{T}^{\Phi}[-m-n / 2] .
$$

Proof. By construction, the operators $\boldsymbol{\Delta}_{\alpha}$ preserve tensor type (with respect to pointwise $\mathrm{SO}(\boldsymbol{h})$ tensor decompositions) and lower homogeneity weight by 2 . Hence $\boldsymbol{\Delta}_{\alpha}^{m}$ maps $\mathcal{T}^{\Phi}(m-n / 2)$ to $\mathcal{T}^{\Phi}(-m-n / 2)$.

To show that $\boldsymbol{\Delta}_{\alpha}^{m}$ acts tangentially, we calculate $\boldsymbol{\Delta}_{\alpha}^{m} Q A$ for $A$ of homogeneity $m-2-n / 2$. Without any homogeneity assumption, we have

$$
\left[\boldsymbol{\Delta}_{\alpha}^{m}, Q\right]=\sum_{p=0}^{m-1} \boldsymbol{\Delta}_{\alpha}^{m-1-p}\left[\boldsymbol{\Delta}_{\alpha}, Q\right] \boldsymbol{\Delta}_{\alpha}^{p} .
$$

Acting on $\mathcal{T}^{\Phi}(w)$, the $p$-th term on the right acts as $2(2(w-2 p)+n+2) \Delta_{\alpha}^{m-1}$ by (25). Hence $\left[\boldsymbol{\Delta}_{\alpha}^{m}, Q\right]$ acts as $2 m(2 w-2 m+n+4) \boldsymbol{\Delta}_{\alpha}^{m-1}$. This vanishes identically if $w=m-2-n / 2$. Thus $\boldsymbol{\Delta}_{\alpha}^{m}$ is tangential on $\boldsymbol{T}^{\Phi}(m-n / 2)$ as desired.

The remainder of this section is concerned with obtaining tractor formulae for the operators in the previous theorem. A key idea is to assume that the ambient tensor field being acted on is suitably "harmonic" as in the following lemma. Since tangential operators do not depend on how the field is extended off 2 , this involves no loss of generality.

Lemma 4.9. Suppose $k \geq 2$ is an integer. In the generic $n$-even case, suppose $k \leq n / 2-1$ or that $\alpha=0$ and $k \leq n / 2$. Given $S \in \mathcal{T}^{\Phi}(k-n / 2)$, suppose $\boldsymbol{\Delta}_{\alpha} S$ is $O\left(Q^{k-1}\right)$. Finally, let $v, 0 \leq v \leq k-1$, be given. Then there is a linear differential operator $\mathscr{P}$ of order at most $2 v$ given by a partial contraction formula polynomial in $\boldsymbol{X}_{A}, \boldsymbol{D}_{A}, \boldsymbol{R}_{A B C D}, \boldsymbol{h}_{A B}$, and $\boldsymbol{h}^{A B}$, such that

$$
\nabla^{v} S=\mathscr{P} S+O\left(Q^{k-v}\right) .
$$

If, on the left-hand side of (46), $S$ has any free indices, then in every term of $\mathscr{P} S$, each of them appears either on $S$ in its natural position or on $\boldsymbol{R}$.

Proof. We will assume that $n$ is even and $M$ is generic. For $v=1$, observe that by (14) and (23) we have

$$
2(k-1) \nabla S=\boldsymbol{D} S-\alpha \boldsymbol{X} \boldsymbol{R} \sharp \sharp S+\boldsymbol{X} \boldsymbol{\Delta}_{\alpha} S .
$$


This is in the required form, since $\boldsymbol{\Delta}_{\alpha} S=O\left(Q^{k-1}\right)$.

We now proceed by induction on $v$. Suppose that $1 \leq m<k-1$ and that (46) holds for $1 \leq v \leq m$. By (14) it follows that

$$
2(k-m-1) \nabla^{m+1} S=\boldsymbol{D} \nabla^{m} S-\alpha \boldsymbol{X} \boldsymbol{R} \sharp \sharp \nabla^{m} S+\boldsymbol{X} \boldsymbol{\Delta}_{\alpha} \nabla^{m} S .
$$

If $S$ on the left-hand side has any free indices, then in every term of the righthand side, each of these indices appears on an $S$ in its natural position or on an $\boldsymbol{R}$. From the inductive assumption and the properties of $\boldsymbol{D}$, it then follows that $\boldsymbol{D} \nabla^{m} S-\alpha \boldsymbol{X} \boldsymbol{R} \sharp \sharp \nabla^{m} S$ is of the form $\mathscr{P} S+O\left(Q^{k-(m+1)}\right)$, where $\mathscr{P}$ is as described in the statement of the lemma. On the other hand, by (42),

$$
\begin{aligned}
\boldsymbol{\Delta}_{\alpha} \nabla^{m} S=\nabla^{m} \boldsymbol{\Delta}_{\alpha} S+\sum\left(\nabla^{p} \boldsymbol{R}\right) & \left(\nabla^{q} S\right) \\
+ & \alpha \sum\left(\nabla^{p+1} \boldsymbol{R}\right)\left(\nabla^{q-1} S\right)+O\left(Q^{n / 2-2-(m-1)}\right),
\end{aligned}
$$

where $p+q=m, p \geq 0$, and $q \geq 1$. When we use (42) to construct (48), each index attached to $S$ on the left-hand side of (48) either remains fixed or moves onto an $\boldsymbol{R}$. Note that $\nabla^{m} \boldsymbol{\Delta}_{\alpha} S$ is $O\left(Q^{k-(m+1)}\right)$ and that $n / 2-2-(m-1) \geq k-(m+1)$. Thus $\boldsymbol{\Delta}_{\alpha} \nabla^{m} S=\sum\left(\nabla^{x} \boldsymbol{R}\right)\left(\nabla^{y} S\right)+O\left(Q^{k-(m+1)}\right)$. Here $x+y=m, x \leq m$, and $y \leq m$. If $\alpha=0$, then we have $1 \leq y$ and $x \leq m-1$. By Theorem 4.7 and by our inductive assumption, it follows that $\Delta_{\alpha} \nabla^{m} S=\mathscr{P} S+O\left(Q^{k-(m+1)}\right)$, where $\mathscr{P}$ is as in the statement of the lemma.

The usefulness of Lemma 4.9 results from the next proposition, which generalises to ambient tensors and $\boldsymbol{\Delta}_{\alpha}$-Laplacians a result of [Graham et al. 1992].

Proposition 4.10. Let $k \geq 1$ be an integer. Then for any $T \in \mathcal{T}^{\Phi}(k-n / 2)$, there is an $S \in \mathcal{T}^{\Phi}(k-n / 2)$ such that $T-S$ is $O(Q)$ and $\boldsymbol{\Delta}_{\alpha} S$ is $O\left(Q^{k-1}\right)$.

Proof. Let $w:=k-n / 2$. Suppose that $S_{m-1} \in \mathcal{T}^{\Phi}(w)$ is such that $T-S_{m-1}$ is $O(Q)$ and $\boldsymbol{\Delta}_{\alpha} S_{m-1}=Q^{m-1} E$. (Then $E \in \mathcal{T}^{\Phi}(w-2 m)$.) If $A \in \mathcal{T}^{\Phi}(w-2 m)$, then $S_{m}:=S_{m-1}+Q^{m} A \in \mathcal{T}^{\Phi}(w)$ and $T-S_{m}$ is $O(Q)$. We have $\boldsymbol{\Delta}_{\alpha} S_{m}=$ $Q^{m-1} E+\boldsymbol{\Delta}_{\alpha} Q^{m} A$. Now $\boldsymbol{\Delta}_{\alpha} Q^{m} A=\sum_{i=0}^{m-1} Q^{i}\left[\boldsymbol{\Delta}_{\alpha}, Q\right] Q^{m-i-1} A+O\left(Q^{m}\right)$, and from (25) and the homogeneity of $A$ and $Q$ this becomes

$$
\begin{aligned}
\boldsymbol{\Delta}_{\alpha} Q^{m} A & =\sum_{i=0}^{m-1} 2(n+2 w-4 i-2) Q^{m-1} A+O\left(Q^{m}\right) \\
& =4 m(w+n / 2-m) Q^{m-1} A+O\left(Q^{m}\right) .
\end{aligned}
$$

Thus if $m \neq w+n / 2$ (i.e. $m \neq k$ ), then setting $A=-[4 m(w+n / 2-m)]^{-1} E$ gives $\boldsymbol{\Delta}_{\alpha} S_{m}=O\left(Q^{m}\right)$.

Note that the proof establishes much more than we require in the proposition. It shows that the $\boldsymbol{\Delta}_{\alpha}$-harmonic extension of $\left.T\right|_{2}$ only fails at $O\left(Q^{k}\right)$, and that past this the extension continues. Also, if we allow $w$ such that $w+n / 2 \notin\{1,2, \ldots\}$, 
then for any $T \in \mathcal{T}^{\Phi}(w)$ and any integer $\ell \geq 0$, there is $S \in \mathcal{T}^{\Phi}(w)$ such that $T-S$ is $O(Q)$ and $\boldsymbol{\Delta}_{\alpha} S$ is $O\left(Q^{\ell}\right)$.

Remark. Recall that one of our central aims (at least for $n \geq 6$ ) is to understand the result of applying $\boldsymbol{\Delta}^{n / 2-2}$ to the ambient curvature $\boldsymbol{R}$. For this it would appear that we do not need Proposition 4.10, since by (22), the ambient curvature already has the property we require of $S$, namely, that $\boldsymbol{\Delta}_{1 / 2} \boldsymbol{R}=\boldsymbol{\Delta} \boldsymbol{R}=O\left(Q^{n / 2-3}\right)$. On the other hand, we prefer here to treat $\boldsymbol{\Delta}^{n / 2-2} \boldsymbol{R}$ in two steps. First, we derive a tractor formula for the conformally invariant operator $\square_{n / 2-2}$ on $\mathscr{T}^{2,2}[-2]$. For this we will use Proposition 4.10. This operator arises from $\boldsymbol{\phi}^{n / 2-2}$ on $\mathcal{T}^{2,2}(-2)$. Then finally we may apply the operator $\square_{n / 2-2}$ to the tractor field $W$; see (28). Proceeding in this way, we can be sure that the tractor formula that we obtain for the ambient quantity $\left.\boldsymbol{A}^{n / 2-2} \boldsymbol{R}\right|_{2}$ is precisely the tractor formula for $\square_{n / 2-2}$ on $\mathscr{T}^{2,2}[-2]$ applied to $W$.

Next, we need to understand how powers of the $\boldsymbol{\Delta}_{\alpha}$-Laplacian are related to iterations of $\boldsymbol{D}$. We begin with a lemma which indicates the impact of moving Laplacians to the right of $\nabla$ 's.

Lemma 4.11. Suppose that $n$ is even and $M$ is generic. Let $\alpha \in \mathbb{R}, w \in \mathbb{R}$, and $T \in \mathcal{T}^{\Phi}(w)$ be given. Let

$$
S=\Delta_{\alpha}^{t_{1}} \nabla^{u_{1}} \cdots \Delta_{\alpha}^{t_{p}} \nabla^{u_{p}} T
$$

where $t_{i}+u_{i} \geq 1$ for each $i$. Suppose that $k:=\sum_{i=1}^{p}\left(t_{i}+u_{i}\right) \leq n / 2-1$. Then

$$
S=\sum\left(\nabla^{v_{1}} \boldsymbol{\Delta}_{\alpha}^{w_{1}} \boldsymbol{R}\right) \cdots\left(\nabla^{v_{q}} \boldsymbol{\Delta}_{\alpha}^{w_{q}} \boldsymbol{R}\right)\left(\nabla^{v_{q+1}} \boldsymbol{\Delta}_{\alpha}^{w_{q+1}} T\right)+O\left(Q^{n / 2-k}\right),
$$

where $v_{j}+w_{j} \leq k$ for each $j$. If $T$ has any free indices in (49), then in (50) these indices appear either on $T$ in their original position or on an $\boldsymbol{R}$.

Proof. We proceed by induction on $k$. Suppose that $1 \leq m \leq n / 2-2$. Suppose the result holds whenever $1 \leq k \leq m$, and let $S$ be as in (49) with $k=m+1$. If $t_{1}=0$, then by our inductive assumption we see immediately that (50) holds modulo $O\left(Q^{n / 2-(m+1)}\right)$. On the other hand, suppose $t_{1}>0$. Then by our inductive assumption, $S=\boldsymbol{\Delta}_{\alpha}\left(\sum\left(\nabla^{v_{1}} \boldsymbol{\Delta}_{\alpha}^{w_{1}} \boldsymbol{R}\right) \cdots\left(\nabla^{v_{q}} \boldsymbol{\Delta}_{\alpha}^{w_{q}} \boldsymbol{R}\right)\left(\nabla^{v_{q+1}} \boldsymbol{\Delta}_{\alpha}^{w_{q+1}} T\right)+O\left(Q^{n / 2-m}\right)\right)$, where $v_{j}+w_{j} \leq m$ for each $j$. Suppose we use the Leibniz rule to expand $\boldsymbol{\Delta}\left(\nabla^{v_{1}} \boldsymbol{\Delta}_{\alpha}^{w_{1}} \boldsymbol{R}\right) \cdots\left(\nabla^{v_{q}} \boldsymbol{\Delta}_{\alpha}^{w_{q}} \boldsymbol{R}\right)\left(\nabla^{v_{q+1}} \boldsymbol{\Delta}_{\alpha}^{w_{q+1}} T\right)$. Then each term in the resulting sum will either contain two factors of the form $\nabla^{v_{j}+1} \boldsymbol{\Delta}_{\alpha}^{w_{j}} \boldsymbol{P}$ or one factor of the form $\boldsymbol{\Delta} \boldsymbol{\nabla}^{v_{j}} \boldsymbol{\Delta}_{\alpha}^{w_{j}} \boldsymbol{P}$, where $\boldsymbol{P}$ denotes $\boldsymbol{R}$ or $T$ in each case. But $\boldsymbol{\Delta} \boldsymbol{\nabla}^{v_{j}} \boldsymbol{\Delta}_{\alpha}^{w_{j}} \boldsymbol{P}$ equals $\boldsymbol{\Delta}_{\alpha} \boldsymbol{\nabla}^{v_{j}} \boldsymbol{\Delta}_{\alpha}^{w_{j}} \boldsymbol{P}-\alpha \boldsymbol{R} \sharp \sharp \nabla^{v_{j}} \boldsymbol{\Delta}_{\alpha}^{w_{j}} \boldsymbol{P}$, and by (42) we may write $\boldsymbol{\Delta}_{\alpha} \boldsymbol{\nabla}^{v_{j}} \boldsymbol{\Delta}_{\alpha}^{w_{j}} \boldsymbol{P}$ in the form $\nabla^{v_{j}} \boldsymbol{\Delta}_{\alpha}^{w_{j}+1} \boldsymbol{P}+\sum\left(\nabla^{v_{\ell}^{\prime}} \boldsymbol{R}\right) \nabla^{v_{\ell}^{\prime \prime}} \boldsymbol{\Delta}_{\alpha}^{w_{j}} \boldsymbol{P}+O\left(Q^{n / 2-(m+1)}\right)$. Here $v_{\ell}^{\prime}+v_{\ell}^{\prime \prime}=v_{j}$. When we use (42), any given index attached to $\boldsymbol{P}$ either remains fixed or moves onto an $\boldsymbol{R}$. This completes the induction. 
Lemma 4.12. Suppose $\ell$ is an integer and $\ell \geq 1$. In the generic $n$-even case, suppose also that $\ell \leq n / 2-1$. Let $T \in \mathcal{T}^{\Phi}(\ell-n / 2)$ be given. Then

$$
\begin{aligned}
\boldsymbol{\Delta}_{\alpha}^{\ell-1} \boldsymbol{D} T=-\boldsymbol{X} & \boldsymbol{\Delta}_{\alpha}^{\ell} T+\sum\left(\nabla^{v_{1}} \boldsymbol{\Delta}^{w_{1}} \boldsymbol{R}\right) \cdots\left(\nabla^{v_{p}} \boldsymbol{\Delta}^{w_{p}} \boldsymbol{R}\right)\left(\nabla^{v_{p+1}} \boldsymbol{\Delta}_{\alpha}^{w_{p+1}} T\right) \\
& +\alpha \boldsymbol{X} \sum\left(\nabla^{r_{1}} \boldsymbol{\Delta}^{s_{1}} \boldsymbol{R}\right) \cdots\left(\nabla^{r_{q}} \boldsymbol{\Delta}^{s_{q}} \boldsymbol{R}\right)\left(\nabla^{r_{q+1}} \boldsymbol{\Delta}_{\alpha}^{s_{q+1}} T\right)+O(Q) .
\end{aligned}
$$

Here $v_{i}+w_{i} \leq \ell-1$ for $1 \leq i \leq p+1$, and $r_{i}+s_{i} \leq \ell-1$ for $1 \leq i \leq q+1$. If $\alpha=0$, then $v_{i}+w_{i} \leq \ell-2$ for $1 \leq i \leq p$, and $v_{p+1}+w_{p+1} \leq \ell-1$. If $T$ on the left-hand side has any free indices, then on the right-hand side these indices always appear on $\boldsymbol{R}$ or in their natural positions on $T$.

Proof. Suppose that $n$ is even and $M$ is generic. If $\ell=1$, the result follows from (14). Now suppose that $\ell \geq 2$. From (14) and (24) we have

$$
\begin{aligned}
& \boldsymbol{\Delta}_{\alpha}^{\ell-1} \boldsymbol{D}_{A} T=2(\ell-1) \Delta_{\alpha}^{\ell-1} \nabla_{A} T-\Delta_{\alpha}^{\ell-1} \boldsymbol{X}_{A} \boldsymbol{\Delta}_{\alpha} T+\alpha \boldsymbol{\Delta}_{\alpha}^{\ell-1} \boldsymbol{X}_{A} \boldsymbol{R} \sharp \sharp T \\
& =2(\ell-1) \Delta_{\alpha}^{\ell-1} \nabla_{A} T-\left[\Delta_{\alpha}^{\ell-1}, \boldsymbol{X}_{A}\right] \boldsymbol{\Delta}_{\alpha} T-\boldsymbol{X}_{A} \Delta_{\alpha}^{\ell-1} \boldsymbol{\Delta}_{\alpha} T \\
& +\alpha\left[\boldsymbol{\Delta}_{\alpha}^{\ell-1}, \boldsymbol{X}_{A}\right] \boldsymbol{R} \sharp \sharp T+\alpha \boldsymbol{X}_{A} \boldsymbol{\Delta}_{\alpha}^{\ell-1} \boldsymbol{R} \sharp \sharp T \\
& =-\boldsymbol{X}_{A} \boldsymbol{\Delta}_{\alpha}^{\ell} T+2(\ell-1) \boldsymbol{\Delta}_{\alpha}^{\ell-1} \nabla_{A} T-\sum_{i=0}^{\ell-2} \boldsymbol{\Delta}_{\alpha}^{\ell-2-i}\left[\boldsymbol{\Delta}_{\alpha}, \boldsymbol{X}_{A}\right] \boldsymbol{\Delta}_{\alpha}^{i} \boldsymbol{\Delta}_{\alpha} T \\
& +\alpha\left(\sum_{i=0}^{\ell-2} \boldsymbol{\Delta}_{\alpha}^{\ell-2-i}\left[\boldsymbol{\Delta}_{\alpha}, \boldsymbol{X}_{A}\right] \boldsymbol{\Delta}_{\alpha}^{i}\right) \boldsymbol{R} \sharp \sharp T+\alpha \boldsymbol{X}_{A} \boldsymbol{\Delta}_{\alpha}^{\ell-1} \boldsymbol{R} \sharp \sharp T \\
& =-\boldsymbol{X}_{A} \boldsymbol{\Delta}_{\alpha}^{\ell} T+2(\ell-1) \boldsymbol{\Delta}_{\alpha}^{\ell-1} \nabla_{A} T-2 \sum_{i=0}^{\ell-2} \Delta_{\alpha}^{\ell-2-i} \nabla_{A} \Delta_{\alpha}^{i} \Delta_{\alpha} T \\
& +2 \alpha\left(\sum_{i=0}^{\ell-2} \Delta_{\alpha}^{\ell-2-i} \nabla_{A} \Delta_{\alpha}^{i}\right) \boldsymbol{R} \sharp \sharp T+\alpha \boldsymbol{X}_{A} \Delta_{\alpha}^{\ell-1} \boldsymbol{R} \sharp \sharp T .
\end{aligned}
$$

Each of the original indices on $T$ remains fixed in this calculation except in the terms of $\boldsymbol{R} \sharp \sharp T$, where it may either remain in its original position on $T$ or move onto an $\boldsymbol{R}$. By (42), we may reexpress this in the form

$$
\begin{aligned}
\boldsymbol{\Delta}_{\alpha}^{\ell-1} \boldsymbol{D} T=-\boldsymbol{X} \boldsymbol{\Delta}_{\alpha}^{\ell} T & +\sum \boldsymbol{\Delta}_{\alpha}^{s_{j}} \boldsymbol{R} \nabla \boldsymbol{\Delta}_{\alpha}^{t_{j}} T+\alpha \sum \boldsymbol{\Delta}_{\alpha}^{s_{j}}(\boldsymbol{\nabla} \boldsymbol{R}) \boldsymbol{\Delta}_{\alpha}^{t_{j}} T \\
& +\alpha \boldsymbol{\Delta}_{\alpha}^{\ell-2} \nabla \boldsymbol{R} \sharp \sharp T+\alpha \sum \boldsymbol{\Delta}_{\alpha}^{p_{i}} \boldsymbol{R} \boldsymbol{\nabla} \boldsymbol{\Delta}_{\alpha}^{q_{i}} \boldsymbol{R} \sharp \sharp T \\
& +\alpha \sum \boldsymbol{\Delta}_{\alpha}^{p_{i}}(\boldsymbol{\nabla} \boldsymbol{R}) \boldsymbol{\Delta}_{\alpha}^{q_{i}} \boldsymbol{R} \sharp \sharp T+\alpha \boldsymbol{X} \boldsymbol{\Delta}_{\alpha}^{\ell-1} \boldsymbol{R} \sharp \sharp T+O(Q),
\end{aligned}
$$

where $s_{j}+t_{j}=\ell-2$ for each $j$ and $p_{i}+q_{i}=\ell-3$ for each $i$. When we use (42) to construct (52), each index on $T$ or $\boldsymbol{R}$ either remains fixed or moves onto an $\boldsymbol{R}$. In the right-hand side of (52) the coefficient of $\boldsymbol{X} \boldsymbol{\Delta}_{\alpha}^{\ell} T$ is exact. Otherwise, no attempt has been made to present the coefficients precisely. At this point we need only the general form of the expression. Where $\alpha$ appears as a coefficient, this means as usual that all terms of this form have coefficient a multiple of $\alpha$.

For ambient tensors $U$ and $V$,

$$
\boldsymbol{\Delta}_{\alpha} U V=(\boldsymbol{\Delta} U) V+(\nabla U) \nabla V+U \boldsymbol{\Delta}_{\alpha} V+\boldsymbol{R} U V .
$$

Thus by using the definition of $\boldsymbol{\Delta}_{\alpha}$ together with the Leibniz rule, we may reexpress the right-hand side of (52) in the form given on the right-hand side of (51), except 
that on each $\boldsymbol{R}$ or $T$, the operators $\nabla, \boldsymbol{\Delta}$, and $\boldsymbol{\Delta}_{\alpha}$ may not be in the order given in (51). But by Lemma 4.11, we may indeed reexpress the right-hand side of (52) in the form given on the right-hand side of (51). In doing this, we may move an index that was originally attached to an $\boldsymbol{R}$ or a $T$, but we always move the index onto an $\boldsymbol{R}$. In the new expression, we have $v_{i}+w_{i} \leq \ell-1$ for $1 \leq i \leq p+1$ and $r_{i}+s_{i} \leq \ell-1$ for $1 \leq i \leq q+1$; this follows from Lemma 4.11. In the $\alpha=0$ case, the fact that $v_{i}+w_{i} \leq \ell-2$ for $1 \leq i \leq p$ follows from the fact that (52) simplifies to $\boldsymbol{\Delta}^{\ell-1} \boldsymbol{D} T=-\boldsymbol{X} \boldsymbol{\Delta}^{\ell} T+\sum \boldsymbol{\Delta}^{s_{j}} \boldsymbol{R} \nabla \boldsymbol{\Delta}^{t_{j}} T+O(Q)$ when $\alpha=0$.

We are now ready to show that the powers of the $\boldsymbol{\Delta}_{\alpha}$-Laplacian can be reexpressed as a sum of compositions of tangential operators.

Proposition 4.13. Suppose $k \geq 1$ is an integer. Let $w=k-n / 2$, and let $V \in \mathcal{T}^{\Phi}(w)$ be given. In the generic n-even case, suppose that $k \leq n / 2-2$, or $\alpha=0$ and $k \leq n / 2-1$, or $\mathcal{T}^{\Phi}(w)=\mathcal{T}(w)$ and $k \leq n / 2-1$, or $\mathcal{T}^{\Phi}(w)=\mathcal{T}^{0}(w)$ and $k \leq n / 2$. Then

$$
(-1)^{k-1} \boldsymbol{X}_{A_{1}} \cdots \boldsymbol{X}_{A_{k-1}} \boldsymbol{\Delta}_{\alpha}^{k} V=\boldsymbol{\Delta} \boldsymbol{D}_{A_{1}} \cdots \boldsymbol{D}_{A_{k-1}} V+\mathscr{P} V+O(Q),
$$

where $\mathscr{P}$ is a linear differential operator of order less than $2 k$ given as a partial contraction polynomial in $\boldsymbol{X}_{A}, \boldsymbol{D}_{A}, \boldsymbol{R}_{A B C D}, \boldsymbol{h}_{A B}$, and $\boldsymbol{h}^{A B}$. If $V$ has any free indices, then for every term of $\mathscr{P} V$, these indices appear either on $\boldsymbol{R}$ or in their natural position on $V$. The indices $A_{i}$ are not skew-symmetrised.

Proof. The case of $V \in \mathcal{T}^{0}(w)$ is treated in [Gover and Peterson 2003]. For the remaining cases, we assume, as usual, that we are in the generic $n$-even setting.

We begin with the case $k \leq n / 2-2$ and the case $\alpha=0$ and $k \leq n / 2-1$; we use induction on $k$. Suppose that $1 \leq m \leq n / 2-3$ or that $\alpha=0$ and $1 \leq m \leq n / 2-2$, and suppose the result holds whenever $k=m$. Let $V \in \mathcal{T}^{\Phi}(m+1-n / 2)$. By Proposition 4.10, there exists an $S \in \mathcal{T}^{\Phi}(m+1-n / 2)$ such that $V-S$ is $O(Q)$ and $\boldsymbol{\Delta}_{\alpha} S$ is $O\left(Q^{m}\right)$. Then by our inductive assumption,

$$
(-1)^{m-1} \boldsymbol{X}_{A_{1}} \cdots \boldsymbol{X}_{A_{m-1}} \boldsymbol{\Delta}_{\alpha}^{m}\left(\boldsymbol{D}_{A_{m}} S\right)=\boldsymbol{\Delta} \boldsymbol{D}_{A_{1}} \cdots \boldsymbol{D}_{A_{m-1}}\left(\boldsymbol{D}_{A_{m}} S\right)+\mathscr{P} S+O(Q),
$$

where $\mathscr{P}$ is of order less than $2 m$. If $S$ on the left-hand side of (53) has any free indices, then in each term of $\mathscr{P} S$, these indices appear either on $\boldsymbol{R}$ or in their natural position on $S$. Now apply Lemma 4.12 with $\ell=m+1$ and $T=S$. We find that

$$
\begin{aligned}
\boldsymbol{\Delta}_{\alpha}^{m} \boldsymbol{D}_{A_{m}} S=-\boldsymbol{X}_{A_{m}} \boldsymbol{\Delta}_{\alpha}^{m+1} S & +O(Q) \\
& +\sum\left(\nabla^{v_{1}} \boldsymbol{\Delta}^{w_{1}} \boldsymbol{R}\right) \cdots\left(\nabla^{v_{p}} \boldsymbol{\Delta}^{w_{p}} \boldsymbol{R}\right)\left(\nabla^{v_{p+1}} \boldsymbol{\Delta}_{\alpha}^{w_{p+1}} S\right) \\
& +\alpha \boldsymbol{X} \sum\left(\nabla^{r_{1}} \boldsymbol{\Delta}^{t_{1}} \boldsymbol{R}\right) \cdots\left(\nabla^{r_{q}} \boldsymbol{\Delta}^{t_{q}} \boldsymbol{R}\right)\left(\nabla^{r_{q+1}} \boldsymbol{\Delta}_{\alpha}^{t_{q+1}} S\right) .
\end{aligned}
$$

Here $v_{i}+w_{i} \leq m$ for $1 \leq i \leq p+1$, and $r_{i}+t_{i} \leq m$ for $1 \leq i \leq q+1$. If $\alpha=0$, then $v_{i}+w_{i} \leq m-1$ for $1 \leq i \leq p$ and $v_{p+1}+w_{p+1} \leq m$. If, on the left-hand side 
of (54), $S$ has any free indices, then on the right-hand side of this equation these indices appear on $\boldsymbol{R}$ or in their natural positions on $S$. Since $\boldsymbol{\Delta}_{\alpha} S$ is $O\left(Q^{m}\right)$, we may assume that $w_{p+1}=t_{q+1}=0$ in (54). Thus by Theorem 4.7 and Lemma 4.9, we have

$$
\boldsymbol{\Delta}_{\alpha}^{m} \boldsymbol{D}_{A_{m}} S=-\boldsymbol{X}_{A_{m}} \Delta_{\alpha}^{m+1} S+\mathscr{P} S+O(Q) .
$$

Since $v_{p+1} \leq m$ and $r_{q+1} \leq m$ in (54), it follows that the order of $\mathscr{P}$ is at most $2 m$ in (55). If $S$ in (55) has free indices, then in $\mathscr{P} S$ these appear either on $\boldsymbol{R}$ or in their natural positions on $S$. From (53) and (55) it now follows that

$$
(-1)^{m} \boldsymbol{X}_{A_{1}} \cdots \boldsymbol{X}_{A_{m}} \boldsymbol{\Delta}_{\alpha}^{m+1} S=\boldsymbol{\Delta} \boldsymbol{D}_{A_{1}} \cdots \boldsymbol{D}_{A_{m}} S+\mathscr{P} S+O(Q) .
$$

But $\boldsymbol{D}_{A}$ acts tangentially along 2, and $\boldsymbol{\Delta}$ acts tangentially on fields homogeneous of degree $1-n / 2$. Thus $\Delta \boldsymbol{D}_{A_{1}} \cdots \boldsymbol{D}_{A_{m}}+\mathscr{P}$ acts tangentially on $S$. By Proposition 4.8, $\boldsymbol{\Delta}_{\alpha}^{m+1}$ also acts tangentially on $S$, and so we may replace $S$ with $V$ on both sides of (56). This completes the induction.

Finally, suppose that $\mathcal{T}^{\Phi}(w)=\mathcal{T}(w)$. By the Ricci-flatness of the ambient metric, it follows that $\boldsymbol{R} \sharp \sharp V$ is $O\left(Q^{n / 2-1}\right)$. Thus for $1 \leq k \leq n / 2-1$ we see that $\Delta_{\alpha}^{k} V=\Delta^{k} V+O(Q)$, and the result follows from the case $\alpha=0$.

We are now ready to prove Theorem 4.1 and at the same time describe tractor formulae for the operators $\square_{m}^{\alpha}$. We begin with the tractor formulae.

Theorem 4.14. Via the algorithm implicit in the inductive steps above, the operators $\square_{m}^{\alpha}$ have tractor formulae (for $m$ in the ranges given in Theorem 4.1) as follows:

$$
(-1)^{m-1} X_{A_{1}} \cdots X_{A_{m-1}} \square_{m}^{\alpha} U=\square D_{A_{1}} \cdots D_{A_{m-1}} U+\mathscr{P}_{A_{1} \cdots A_{m-1}}^{\Phi, m} U,
$$

where the differential operator $\mathscr{P}^{\Phi, m}$ is a partial contraction polynomial in $X, D$, $W, h$, and $h^{-1}$. Thus for $m \neq n / 2$,

$$
\begin{aligned}
(m-1) ! & \left(\prod_{i=2}^{m}(n-2 i)\right) \square_{m}^{\alpha} U \\
& =D^{A_{m-1}} \cdots D^{A_{1}} \square D_{A_{1}} \cdots D_{A_{m-1}} U+D^{A_{m-1}} \cdots D^{A_{1} \mathscr{P}_{A_{1} \cdots A_{m-1}}^{\Phi, m}} U .
\end{aligned}
$$

The indices attached to $U$ on the left-hand side appear, in each term of $\mathscr{P}^{\Phi, m} U$, on $U$ in their original position or on $W$. The indices $A_{i}$ in (57) and (58) are not skew-symmetrised.

Proof of theorems 4.1 and 4.14. Recall that $\boldsymbol{\Delta}: \mathcal{T}^{\Phi}(1-n / 2) \rightarrow \mathcal{T}^{\Phi}(-1-n / 2)$ descends to the generalised conformal Laplacian operator $\square$ and $\boldsymbol{D}$ descends to $D$; see (17). Thus (57) is an immediate consequence of Proposition 4.13. From this the claims of naturality are immediate from the naturality of $X, \square, D, W, h$, and $h^{-1}$. That the $\square_{m}^{\alpha}$ have leading term $\Delta^{m}$ follows easily from the expression (16) 
for $D$ and the identities (9) for the tractor connection. Then note that (58) follows from (57) and (19).

4B. Calculating explicit formulae; examples. One can easily compute explicit formulae for the obstruction tensors in low dimensions. From the proof of theorems 4.2 and 4.4, we know that in dimension $4, O_{a b}$ is simply $-(1 / 2) B_{a b}$, where $B_{a b}$ is the Bach tensor as given in (1).

In dimension 6, we have $m=1$, and the relevant ambient operator from Proposition 4.8 is $\boldsymbol{\Delta}: \mathcal{T}^{2,2}(-2) \rightarrow \mathcal{T}^{2,2}(-4)$, which descends to

$$
\square+\frac{1}{4} W \sharp \sharp=: \not_{1}: \mathscr{T}^{2,2}[-2] \rightarrow \mathscr{T}^{2,2}[-4] .
$$

The left-hand side of (59) is the tractor formula for $\square_{1}$. By Theorem 4.2, applying this to $W$ yields the obstruction tensor via the identity (32); see (28). That is, $2^{6} X_{A_{1}} Z_{A_{2}}{ }^{a} X_{B_{1}} Z_{B_{2}}{ }^{b} O_{a b}^{6}=\square W+\frac{1}{4} W \sharp \sharp W$, where we have used the fact that $k(6)=$ $2^{6}$. Thus

$$
\begin{aligned}
640_{C e} X_{[B} Z_{C]}{ }^{c} Z_{[E}^{e} X_{D]} & \\
& =\square W_{B C D E}-W^{A}{ }_{C B}{ }^{F} W_{F A D E}-W^{A}{ }_{C D}{ }^{F} W_{B A F E}-W^{A}{ }_{C E}{ }^{F} W_{B A D F} .
\end{aligned}
$$

But $4 Y^{B} Y^{D} Z^{C}{ }_{a} Z^{E}{ }_{b} X_{[B} B_{C][E} X_{D]}=\mathcal{O}_{a b}$. Thus, in any conformal scale, $\mathscr{O}_{a b}$ is given by

$$
\begin{aligned}
& \frac{1}{16} Y^{B} Y^{D} Z_{a}^{C} Z_{b}^{E} \\
& \quad \times\left(\square W_{B C D E}-W_{C B}^{A}{ }^{F} W_{F A D E}-W_{C D}^{A}{ }^{F} W_{B A F E}-W_{C E}^{A}{ }^{F} W_{B A D F}\right) .
\end{aligned}
$$

If one expands using (9), (28) and the definitions of $\square$ and the tractor metric, it is an entirely mechanical process to rewrite (60) in terms of the Levi-Civita connection and its curvature (with metric contractions). A computation using this process together with Mathematica and J. Lee's Ricci software package [Lee 1998] shows that

$$
\begin{aligned}
\mathrm{O}_{a b}^{6}=\frac{1}{16} \Delta B_{a b} & -\frac{1}{4} J B_{a b}+\frac{1}{8} B_{c d} C_{a}{ }^{c}{ }^{d}-\frac{1}{2} P_{c d} \nabla^{c} A_{(a b)}{ }^{d} \\
& +\frac{1}{4} A_{c a d} A^{c}{ }_{b}{ }^{d}-\frac{1}{2} A_{c a d} A^{d}{ }_{b}{ }^{c}-\frac{1}{4} A_{(a b) c} \nabla^{c} J+\frac{1}{4} P_{c d} P^{d}{ }_{e} C_{a}{ }^{c} b^{e},
\end{aligned}
$$

where $A$ and $B$ are respectively the Cotton and Bach tensors as given in (29) and (30). This formula for $\mathbb{O}_{a b}^{6}$ agrees up to a constant factor with the formula given in [Graham and Hirachi 2005].

In dimension 8 , we find that $O_{a b}=(1 / 384) T_{(a b)}$, where $T_{a b}$ is as given on the next page. To see that $O_{a b}^{8}=\mathrm{T}_{(a b)}$, up to a constant factor, we begin by constructing a tractor formula for $\square_{2}$ on $\mathscr{T}^{2,2}[-2]$. Let $T \in \mathcal{T}^{\Phi}(-2)$ be an extension of any element of $\mathscr{T}^{2,2}[-2]$. By Proposition 4.10 we may assume that $\Delta T=O(Q)$. Thus 


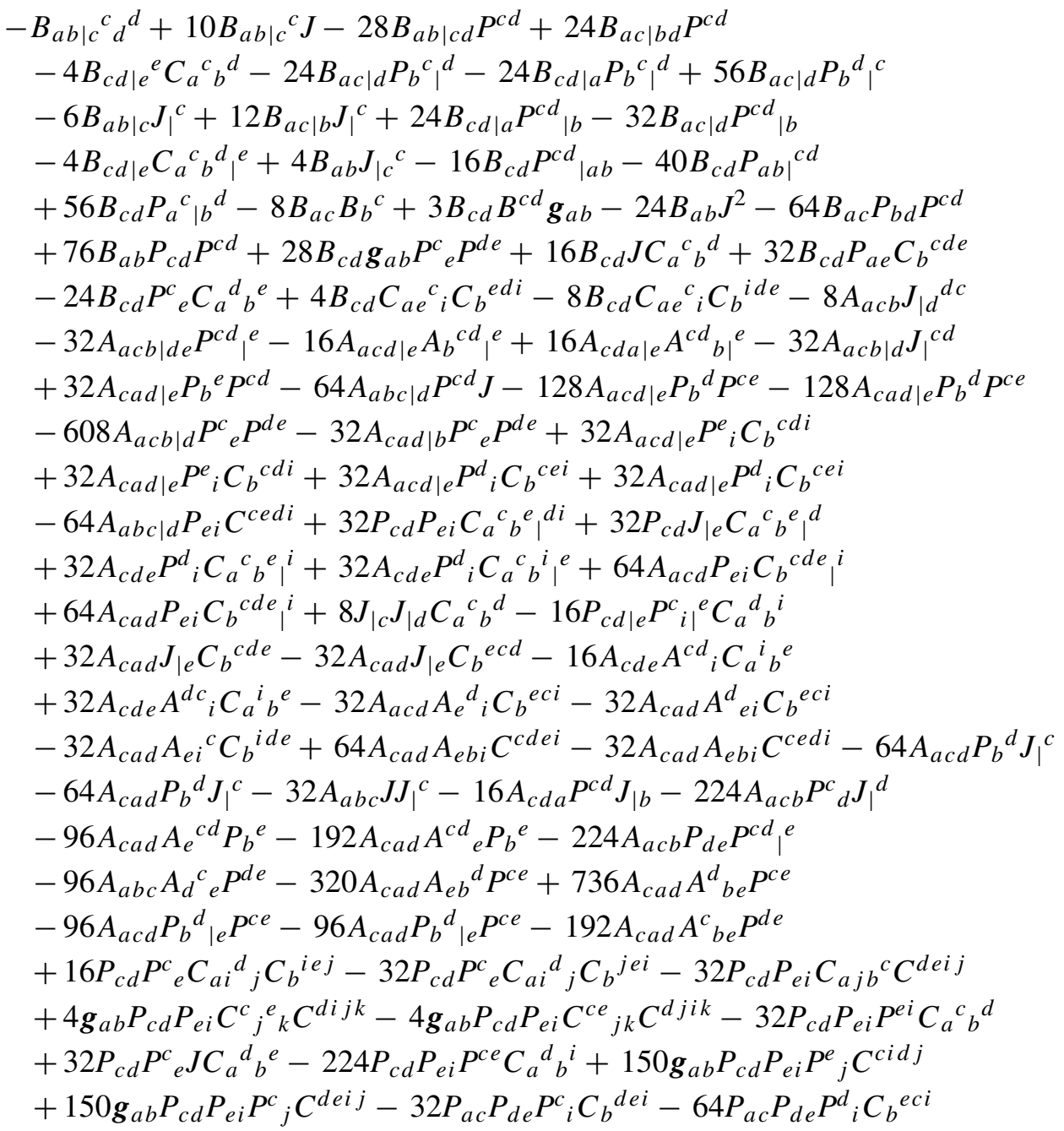

A tensor $\mathrm{T}_{a b}$ such that $\mathcal{O}_{a b}^{8}=\frac{1}{384} \mathrm{~T}_{(a b)}$. Here $B_{a b \mid c d}:=\nabla_{d} \nabla_{c} B_{a b}$, etc.

by (14), (22), and (24), we first obtain

$$
\begin{aligned}
& \boldsymbol{\Delta} \boldsymbol{D}_{A} T_{B C D E}=-\boldsymbol{X}_{A} \boldsymbol{\Delta}^{2} T_{B C D E}+2\left[\boldsymbol{\Delta}, \nabla_{A}\right] T_{B C D E}+\boldsymbol{R} \sharp \sharp \nabla_{A} T_{B C D E} \\
&-\frac{1}{4} \boldsymbol{X}_{A}(\boldsymbol{R} \sharp \sharp \boldsymbol{R}) \sharp \sharp T_{B C D E}+\boldsymbol{X}_{A}\left(\nabla_{|I|} \boldsymbol{R}\right) \sharp \sharp \nabla^{|I|} T_{B C D E}+O(Q) .
\end{aligned}
$$

Here the $|\cdot|$ indicates that the enclosed index is not involved in the hash action, and $(\boldsymbol{R} \sharp \sharp \boldsymbol{R}) \sharp \sharp T_{B C D E}$ denotes the double hash of $\boldsymbol{R} \sharp \sharp \boldsymbol{R}$ with $T_{B C D E \text {. From this }}$ equation together with (5), (14), (22), and (26), it follows that 


$$
\begin{aligned}
\Delta \boldsymbol{D}_{A} T_{B C D E}=-\boldsymbol{X}_{A} \Delta^{2} T_{B C} & -2 \boldsymbol{R}_{A}{ }^{P}{ }_{B}{ }^{Q} \boldsymbol{D}_{P} T_{Q C D E}-2 \boldsymbol{R}_{A}{ }^{P}{ }_{C}{ }^{Q} \boldsymbol{D}_{P} T_{B Q D E} \\
& -2 \boldsymbol{R}_{A}{ }^{P}{ }_{D}{ }^{Q} \boldsymbol{D}_{P} T_{B C Q E}-2 \boldsymbol{R}_{A}{ }^{P}{ }_{E}{ } \boldsymbol{D}_{P} T_{B C D Q} \\
& +\frac{1}{2} \boldsymbol{R} \sharp \sharp \boldsymbol{D}_{A} T_{B C D E}-\frac{1}{4} \boldsymbol{R} \sharp \sharp \boldsymbol{X}_{A} \boldsymbol{R} \sharp \sharp T_{B C D E} \\
& -\frac{1}{4} \boldsymbol{X}_{A}(\boldsymbol{R} \sharp \sharp \boldsymbol{R}) \sharp \sharp T_{B C D E}+\frac{1}{4} \boldsymbol{X}_{A}\left(\boldsymbol{D}_{|I|} \boldsymbol{R}\right) \sharp \sharp \boldsymbol{D}^{|I|} T_{B C D E} \\
& -\frac{1}{8} \boldsymbol{X}_{A} \boldsymbol{X}_{I}(\boldsymbol{R} \sharp \sharp \boldsymbol{R}) \sharp \sharp \boldsymbol{D}^{|I|} T_{B C D E} \\
& -\frac{1}{8} \boldsymbol{X}_{A} \boldsymbol{X}^{I}\left(\boldsymbol{D}_{|I|} \boldsymbol{R}\right) \sharp \sharp \boldsymbol{R} \sharp \sharp T_{B C D E}+O(Q) .
\end{aligned}
$$

Since the dimension is 8, it follows from (14) that $\boldsymbol{X}^{A} \boldsymbol{D}_{A} V=-4 V+O(Q)$ for all $V \in \mathcal{T}^{\Phi}(-2)$. Thus from the definition of $\boldsymbol{\Delta}$ we see that

$$
\begin{aligned}
& \boldsymbol{X}_{A} \boldsymbol{\Delta}^{2} T_{B C D E}=-\boldsymbol{\Delta} \boldsymbol{D}_{A} T_{B C D E}-2 \boldsymbol{R}_{A}{ }^{P}{ }_{B}{ }^{Q} \boldsymbol{D}_{P} T_{Q C D E}-2 \boldsymbol{R}_{A}{ }^{P}{ }_{C}{ }^{Q} \boldsymbol{D}_{P} T_{B Q D E} \\
& -2 \boldsymbol{R}_{A}{ }^{P}{ }_{D}^{Q} \boldsymbol{D}_{P} T_{B C Q E}-2 \boldsymbol{R}_{A}{ }^{P}{ }_{E}^{Q} \boldsymbol{D}_{P} T_{B C D Q} \\
& -\frac{1}{4} \boldsymbol{R} \sharp \sharp \boldsymbol{X}_{A} \boldsymbol{R} \sharp \sharp T_{B C D E}+\frac{1}{4} \boldsymbol{X}_{A}(\boldsymbol{R} \sharp \sharp \boldsymbol{R}) \sharp \sharp T_{B C D E} \\
& +\frac{1}{4} \boldsymbol{X}_{A}\left(\boldsymbol{D}_{|I|} \boldsymbol{R}\right) \sharp \sharp \boldsymbol{D}^{|I|} T_{B C D E}+\frac{1}{2} \boldsymbol{X}_{A} \boldsymbol{R} \sharp \sharp \boldsymbol{R} \sharp \sharp T_{B C D E} \\
& +O(Q) \text {. }
\end{aligned}
$$

We restrict this to 2 and then attach $Y^{A}$. The result is that for any $T \in \mathscr{T}^{2,2}[-2]$,

$$
\begin{aligned}
\square_{2} T_{B C D E}=-Y^{A} \square & D_{A} T_{B C D E}-\frac{1}{2} Y^{A} W_{A}{ }^{P}{ }_{B}{ }^{Q} D_{P} T_{Q C D E} \\
& -\frac{1}{2} Y^{A} W_{A}{ }^{P}{ }_{C}{ }^{Q} D_{P} T_{B Q D E}-\frac{1}{2} Y^{A} W_{A}{ }^{P}{ }_{D}{ }^{Q} D_{P} T_{B C Q E} \\
& -\frac{1}{2} Y^{A} W_{A}{ }^{P}{ }_{E}{ }^{Q} D_{P} T_{B C D Q}-\frac{1}{64} Y^{A} W \sharp \sharp X_{A} W \sharp \sharp T_{B C D E} \\
& +\frac{1}{64}(W \sharp \sharp W) \sharp \sharp T_{B C D E}+\frac{1}{16}\left(D_{|I|} W\right) \sharp \sharp D^{|I|} T_{B C D E} \\
& +\frac{1}{32} W \sharp \sharp W \sharp \sharp T_{B C D E .}
\end{aligned}
$$

We use this to construct a tractor formula for $0_{a b}^{8}$. From Theorem 4.2 we have

$$
\mathrm{O}_{c e}^{8}=-\frac{1}{384} Y^{B} Z^{C}{ }_{c} Y^{D} Z^{E}{ }_{e} \square_{2} W_{B C D E} .
$$

A short computation shows that $W \sharp \sharp W \sharp \sharp W_{B C D E}=(W \sharp \sharp W) \sharp \sharp W_{B C D E}$. Thus from (62) and (63) we have

$$
\begin{aligned}
0_{a b}^{8}=\frac{1}{24576} Y^{B} Z^{C}{ }_{a} & Y^{D} Z^{E}{ }_{b}\left(64 Y^{A} \square D_{A} W_{B C D E}\right. \\
& +32 Y^{A} W_{A}{ }^{P}{ }_{B}{ }^{Q} D_{P} W_{Q C D E}+32 Y^{A} W_{A}{ }^{P}{ }_{C}{ }^{Q} D_{P} W_{B Q D E} \\
& +32 Y^{A} W_{A}{ }^{P}{ }_{D}{ }^{Q} D_{P} W_{B C Q E}+32 Y^{A} W_{A}{ }^{P}{ }_{E}{ }^{Q} D_{P} W_{B C D Q} \\
& +Y^{A} W \sharp \sharp X_{A} W \sharp \sharp W_{B C D E}-3 W \sharp \sharp W \sharp \sharp W_{B C D E} \\
& \left.-4\left(D_{|I|} W\right) \sharp \sharp D^{|I|} W_{B C D E}\right) .
\end{aligned}
$$

By using the same techniques as in our derivation of (61), we see that $\mathbb{O}_{a b}^{8}=\mathrm{T}_{(a b)}$. 


\section{References}

[Anderson 2005] M. T. Anderson, "Orbifold compactness for spaces of Riemannian metrics and applications”, Math. Ann. 331:4 (2005), 739-778. MR 2006c:53029 Zbl 1071.53025

[Bach 1921] R. Bach, "Zur Weylschen Relativitätsth. und der Weylschen Erweiterung des Krümmungstensorbegriffs", Math. Z. 9:1-2 (1921), 110-135. MR MR1544454 JFM 48.1035.01

[Bailey et al. 1994a] T. N. Bailey, M. G. Eastwood, and A. R. Gover, "Thomas's structure bundle for conformal, projective and related structures", Rocky Mountain J. Math. 24:4 (1994), 1191-1217. MR 96e:53016 Zbl 0828.53012

[Bailey et al. 1994b] T. N. Bailey, M. G. Eastwood, and C. R. Graham, "Invariant theory for conformal and CR geometry", Ann. of Math. (2) 139:3 (1994), 491-552. MR 95h:53016 Zbl 0814.53017

[Boe and Collingwood 1985] B. D. Boe and D. H. Collingwood, "A comparison theory for the structure of induced representations", J. Algebra 94 (1985), 511-545. MR 87b:22026a Zbl 0606.17007

[Branson 1995] T. P. Branson, "Sharp inequalities, the functional determinant, and the complementary series”, Trans. Amer. Math. Soc. 347:10 (1995), 3671-3742. MR 96e:58162 Zbl 0848.58047

[Branson 2005] T. Branson, “Q-curvature and spectral invariants", Rend. Circ. Mat. Palermo (2) Suppl. 75 (2005), 11-55. MR 2006h:53024

[Branson and Gover 2001] T. Branson and A. R. Gover, "Conformally invariant non-local operators”, Pacific J. Math. 201:1 (2001), 19-60. MR 2003e:58036 Zbl 1052.58026

[Branson and Gover 2002] T. Branson and A. R. Gover, "Electromagnetism, metric deformations, ellipticity and gauge operators on conformal 4-manifolds", Differential Geom. Appl. 17:2-3 (2002), 229-249. MR 2003i:58036 Zbl 1025.58008

[Branson and Gover 2005] T. Branson and A. R. Gover, "Conformally invariant operators, differential forms, cohomology and a generalisation of $Q$-curvature", Comm. Partial Differential Equations 30:10-12 (2005), 1611-1669. MR MR2182307 Zbl 02238799

[Branson and Gover, in progress] T. Branson and A. R. Gover, "Detour torsion”. In progress.

[Branson and Ørsted 1991] T. P. Branson and B. Ørsted, "Explicit functional determinants in four dimensions", Proc. Amer. Math. Soc. 113:3 (1991), 669-682. MR 92b:58238 Zbl 0762.47019

[Čap and Gover 2000] A. Čap and A. R. Gover, "Tractor bundles for irreducible parabolic geometries", pp. 129-154 in Global analysis and harmonic analysis (Marseille-Luminy, 1999), edited by J. P. Bourguignon et al., Sémin. Congr. 4, Soc. Math. France, Paris, 2000. MR 2002b:53033 Zbl 0996.53012

[Čap and Gover 2003] A. Čap and A. R. Gover, "Standard tractors and the conformal ambient metric construction”, Ann. Global Anal. Geom. 24:3 (2003), 231-259. MR 2004g:53016 Zbl 1039.53021

[Eastwood 1996] M. Eastwood, "Notes on conformal differential geometry", Rendiconti Circ. Mat. Palermo (2) Suppl. 43 (1996), 57-76. MR 98g:53021 Zbl 0911.53020

[Eastwood and Slovák 1997] M. Eastwood and J. Slovák, "Semiholonomic Verma modules", J. Algebra 197:2 (1997), 424-448. MR 98k:22054 Zbl 0907.17010

[Fefferman 1979] C. Fefferman, "Parabolic invariant theory in complex analysis", Adv. in Math. 31:2 (1979), 131-262. MR 80j:32035 Zbl 0444.32013

[Fefferman and Graham 1985] C. Fefferman and C. R. Graham, "Conformal invariants", Astérisque Numero Hors Serie (1985), 95-116. The math. heritage of Élie Cartan (Lyon, 1984). MR 87g:53060 Zbl 0602.53007

[Fefferman and Graham, in progress] C. Fefferman and C. R. Graham, In progress. 
[Gasqui and Goldschmidt 1984] J. Gasqui and H. Goldschmidt, Déformations infinitésimales des structures conformes plates, Progr. in Mathematics 52, Birkhäuser, Boston, 1984. MR 86m:58156 Zbl 0585.53001

[Gauduchon 1990] P. Gauduchon, "Connexion canonique et structures de Weyl en géométrie conforme", Preprint CNRS UA766, Ecole Polytechnique, Paris, 1990.

[Gover 1999] A. R. Gover, "Aspects of parabolic invariant theory", Rend. Circ. Mat. Palermo (2) Suppl. 59 (1999), 25-47. MR 2001a:58047 Zbl 0967.53033

[Gover 2001] A. R. Gover, "Invariant theory and calculus for conformal geometries", Adv. Math. 163:2 (2001), 206-257. MR 2003a:53016 Zbl 1004.53010

[Gover and Hirachi 2004] A. R. Gover and K. Hirachi, "Conformally invariant powers of the Laplacian - a complete nonexistence theorem”, J. Amer. Math. Soc. 17:2 (2004), 389-405. MR 2005c: 58062 Zbl 1066.53037

[Gover and Nurowski 2006] A. R. Gover and P. Nurowski, "Obstructions to conformally Einstein metrics in $n$ dimensions", J. Geom. Phys. 56:3 (2006), 450-484. MR 2171895

[Gover and Peterson 2003] A. R. Gover and L. J. Peterson, "Conformally invariant powers of the Laplacian, $Q$-curvature, and tractor calculus”, Comm. Math. Phys. 235:2 (2003), 339-378. MR 2004d:58047 Zbl 1022.58014

[Graham and Hirachi 2005] C. R. Graham and K. Hirachi, "The ambient obstruction tensor and $Q$ curvature", pp. 59-71 in AdS/CFT correspondence: Einstein metrics and their conformal boundaries, IRMA Lect. Math. Theor. Phys. 8, European Math. Society, Zürich, 2005. MR 2160867 Zbl 1074.53027

[Graham et al. 1992] C. R. Graham, R. Jenne, L. J. Mason, and G. A. J. Sparling, "Conformally invariant powers of the Laplacian. I. Existence", J. London Math. Soc. (2) 46:3 (1992), 557-565. MR 94c:58226 Zbl 0726.53010

[Lee 1998] J. Lee, Ricci (software package), 1998, Available at http://www.math.washington.edu/ $\sim$ lee/Ricci/.

[Thomas 1926] T. Thomas, "On conformal geometry", Proc. Natl. Acad. Sci. USA 12 (1926), 352359. JFM 52.0736.01

[Tian and Viaclovsky 2005] G. Tian and J. Viaclovsky, "Bach-flat asymptotically locally Euclidean metrics”, Invent. Math. 160:2 (2005), 357-415. MR 2006b:53050 Zbl 1085.53030

Received November 4, 2004. Revised March 21, 2005.

\author{
A. Rod Gover \\ DEPARTMENT OF MATHEMATICS \\ The University of AUCKLAND \\ PRIVATE BAG 92019 \\ AUCKLAND 1 \\ New ZeAland \\ gover@math.auckland.ac.nz \\ LAWrence J. Peterson \\ DePaRtMENT OF MATHEMATiCS \\ THE UNIVERSITY OF NORTH DAKOTA \\ GRAND FORKS, ND 58202-8376 \\ USA \\ lawrence.peterson@und.nodak.edu
}

